\title{
A Finite Element Approach for Computing \\ Edge Singularities in Piezoelectric Materials
}

\author{
K.Y.Sze , Hai-tao Wang \\ Department of Mechanical Engineering, The University of Hong Kong \\ Pokfulam Road, Hong Kong, P.R.CHINA
}

and

H.Fan

School of Mechanical and Production Engineering, Nanyang Technological University

Nanyang Avenue, SINGAPORE 639798

\begin{abstract}
By using the eigenfunction expansion technique and the weak form of the governing equations for prismatic sectorial domains composed of piezoelectrics and air, an onedimensional finite element procedure is formulated for computing the eigensolutions of the electromechanical field problem. Generalized displacement and electric potential are taken to be the nodal variables. The resulting global equation is a second order characteristic matrix equation. Validity of the formulation is verified by comparing the computed results with the existing solutions for impermeable cracks and interfacial cracks. Configurations which are of practical interest including conducting cracks, permeable and impermeable notches are studied.
\end{abstract}

keyword : singularity, piezoelectricity, finite element method, crack, eigensolution

Published in Inter.J.Solids \& Structures 38: 9233-9252 (2001). 


\section{INTRODUCTION}

Piezoelectrics have been extensively used in sensors, actuators, resonators and intelligent structures. Reliable service lifetime predictions of piezoelectrics components demand a complete understanding of their fracture processes. In this light, a large amount of analytical works have been devoted to cracks inside homogeneous piezoelectrics, interfacial cracks between dissimilar piezoelectrics and cracks terminated at the interfaces of dissimilar piezoelectrics (Parton 1976; McMeeking 1989; Pak 1990; Sosa \& Pak 1990; Kuo \& Barnett 1991; Sosa 1992; Suo, Kuo, Barnett \& Willis 1992; Dunn 1994; Hao \& Shen 1994; Park \& Sun 1995; Qin \& Yu 1997). Apart from the three conventional modes of fracture characterized by various relative displacement modes of the crack faces, there exits the fourth mode of fracture featured by non-zero electric potential and singular electric field. In most of the cited works, the crack face boundary conditions were often assumed to be traction-free and charge-free. While the traction-free condition is standard for opening cracks, the charge-free assumption has attracted considerable debates. The condition is the intrinsic property of the so-called impermeable cracks through which the electric flux cannot pass. Strong electric field concentration is found at the crack tip and gives rise to the fourth eigensolutions. The impermeable assumption is rationalized by the relative magnitudes of the permittivities of air and piezoceramics. The ratio is of the order $10 \sim 1000$. On the other hand, the crack angle is very small and air is indeed permeable. For a sufficiently small crack angle, it is argued that the air gap or the crack can be practically conducting and the crack faces are equipotential. Needless to say, the permeable condition which takes into account of the crack angle and air permittivities is most realistic. Unfortunately, it poses considerable complications to the analytical treatment and, thus, is least employed. Analytical treatments for conducting and permeable cracks were presented by Dunn (1994) as well as Hao \& Shen (1994).

Identification of the eigensolutions is always the fundamental step for understanding the fracture process. In this context, Sosa \& Pak (1990) employed the eigenfunction expansion technique introduced by Hartranft \& Sih (1969) to study impermeable cracks embedded in transverse isotropic piezoelectrics. With the Stroh's formalism and the notion of surface impedance tensor, Kuo \& Barnett (1991) investigated impermeable and conducting interfacial cracks in bonded dissimiliar piezoelectrics. More recently, Qin \& Yu (1997) also employed the Stroh's formalism to study impermeable cracks terminated at the interfaces of bonded dissimiliar piezoelectrics. To make the derivation tractable, the concept of axial conjugate was introduced.

Like most engineering problems, derivation of analytical eigen-solutions is limited to very simple configurations and requires a high level of mathematical competence. Naturally, numerical methods for determining eigen-solutions on have been proposed. For instances, Yamada \& 
Okumura (1983) employed the virtual work principle and a singular transformation technique with which the displacement of the devised sectorial finite elements become proportional to a certain power of the radial displacement from the crack tip. The same approach was later adopted by Pageau \& Biggers (1996) for bimaterial systems making up of anisotropic materials. Gu \& Belytschko (1994) derived a stress function-based and a displacement-based methods which result respectively in a fourth and a second order characteristic matrix equations. On the other hand, Yosibash \& Szabo (1995) proposed the Steklov methods which again assume the same asymptotic form of the displacement. The methods start with a weak form of the homogeneous equilibrium equations and result in a linear characteristic matrix equations. Sze \& Wang (2000) also devised a simple finite element formulation with which convergence can be yielded either by $h$ - or $p$ refinement. However, all of the above works are restricted to conventional materials and have never been extended to piezoelectric or other reactive materials.

In this paper, the previously devised finite element procedure for eigenfunction analyses of conventional materials (Sze \& Wang 2000) will be generalized to piezoelectric materials. Moreover, air as permeable, impermeable or conducting medium is also considered. Same as most, if not all, analytical works on subject matter, we shall restrict to edge singularities in which the field variables are proportional to certain power of the perpendicular distance from a straight edge. Vertex singularities (see, e.g., Bazant \& Estenssoro 1979, Benthem 1980, Pageau 1995) in which the field variables are proportional to certain power of the distance from a vertex point will not be considered. The computed results are in good agreement with their analytical counterparts if any.

\section{STRONG FORMULATION FOR PERMEABLE NOTCH IN PIEZOELECTRICS}

Piezoelectric effects were first discovered in 1880 by the Curie brothers. The production of an electric potential gradient by mechanically straining a piece of piezoelectric material is called the direct piezoelectric effect. The converse effect, whereby a mechanical strain is produced by subjecting the material to an electric field, also exists. The effects can be expressed as :

$$
\left\{\begin{array}{l}
\boldsymbol{\sigma} \\
\mathbf{D}
\end{array}\right\}=\left[\begin{array}{cc}
\mathbf{C} & -\mathbf{e}^{T} \\
\mathbf{e} & \in
\end{array}\right]\left\{\begin{array}{l}
\boldsymbol{\epsilon} \\
\mathbf{E}
\end{array}\right\}
$$

in which $\boldsymbol{\sigma}, \mathbf{D}, \boldsymbol{\epsilon}$ and $\mathbf{E}$ are the vectors of stress, electric displacement, strain and electric field components, respectively. Moreover, $\mathbf{C}, \mathbf{e}$ and $\in$ are the elasticity, piezoelectric and permittivity matrices, respectively. The electric displacement is, perhaps, best defined by the surface charge density which is the dot product of the electric displacement and the unit outward normal vector to 
the surface.

Figure 1 shows a prismatic sectorial domain in which subdomain $\Omega^{A}$ is an air notch, subdomains $\Omega^{B}$ and $\Omega^{C}$ are piezoelectric materials. Furthermore, air is considered as a permeable medium. Under the cylindrical coordinates $(r-\theta-z)$, the governing equations for the electromechanical eigenanalysis are given below :

(c1) homogeneous stress equilibrium condition :

$$
\left\{\begin{array}{c}
\boldsymbol{\nabla}\left(r \boldsymbol{\sigma}_{r}\right)-\tau_{\theta \theta} \\
\boldsymbol{\nabla}\left(r \boldsymbol{\sigma}_{\theta}\right)+\tau_{r \theta} \\
\boldsymbol{\nabla}\left(r \boldsymbol{\sigma}_{z}\right)
\end{array}\right\}=\boldsymbol{\nabla}_{m}(r \boldsymbol{\sigma})=\mathbf{0} \quad \text { in } \Omega^{B} \text { and } \Omega^{C}
$$

in which

$$
\begin{aligned}
& \boldsymbol{\nabla}=\left\{\frac{\partial}{\partial r}, \frac{1}{r} \frac{\partial}{\partial \theta}, \frac{\partial}{\partial z}\right\}, \boldsymbol{\sigma}_{r}=\left\{\begin{array}{c}
\tau_{r r} \\
\tau_{r \theta} \\
\tau_{r z}
\end{array}\right\}, \boldsymbol{\sigma}_{\theta}=\left\{\begin{array}{c}
\tau_{r \theta} \\
\tau_{\theta \theta} \\
\tau_{\theta z}
\end{array}\right\}, \boldsymbol{\sigma}_{z}=\left\{\begin{array}{c}
\tau_{r z} \\
\left.\tau_{\theta z}\right\} \\
\tau_{z z}
\end{array}\right\}, \\
& \boldsymbol{\sigma}=\left\{\tau_{r r}, \tau_{\theta \theta}, \tau_{z z}, \tau_{\theta z}, \tau_{r z}, \tau_{r \theta}\right\}^{T} \text { and } \nabla_{m} \text { is self-defined. }
\end{aligned}
$$

(c2) charge balance rule :

$$
\boldsymbol{\nabla}(r \mathbf{D})=0 \quad \text { in } \Omega^{A}, \Omega^{B} \text { and } \Omega^{C}
$$

(c3) traction-reciprocity condition :

$$
\left.\left(\boldsymbol{\sigma}_{\theta}^{B}-\boldsymbol{\sigma}_{\theta}^{C}\right)\right|_{\theta=\theta_{B C}}=\mathbf{0}
$$

(c4) traction-free conditions :

$$
\left.\boldsymbol{\sigma}_{\theta}^{B}\right|_{\theta=\theta_{C A}}=\left.\boldsymbol{\sigma}_{\theta}^{C}\right|_{\theta=\theta_{A B}}=\mathbf{0}
$$

(c5) charge-reciprocity conditions :

$$
\left.\left(D_{\theta}^{B}-D_{\theta}^{C}\right)\right|_{\theta=\theta_{B C}}=0,\left.\left(D_{\theta}^{C}-D_{\theta}^{A}\right)\right|_{\theta=\theta_{C A}}=0,\left.\left(D_{\theta}^{A}-D_{\theta}^{B}\right)\right|_{\theta=\theta_{A B}}=0
$$

(c6) strain-displacement relation :

$$
\boldsymbol{\epsilon}=\left\{\frac{\partial u_{r}}{\partial r}, \frac{u_{r}}{r}+\frac{1}{r} \frac{\partial u_{\theta}}{\partial \theta}, \frac{\partial u_{z}}{\partial z}, \frac{\partial u_{\theta}}{\partial z}+\frac{1}{r} \frac{\partial u_{z}}{\partial \theta}, \frac{\partial u_{z}}{\partial r}+\frac{\partial u_{r}}{\partial z}, \frac{1}{r} \frac{\partial u_{r}}{\partial \theta}+r \frac{\partial}{\partial r}\left(\frac{u_{\theta}}{r}\right)\right\}^{T} \quad \text { in } \Omega^{B} \text { and } \Omega^{C}
$$

in which $u_{r}, u_{\theta}$ and $u_{z}$ are the radial, circumferential and longitudinal displacements, respectively. 
(c7) electric field-electric potential relation :

$$
\mathbf{E}=-\boldsymbol{\nabla} \phi \quad \text { in } \Omega^{A}, \Omega^{B} \text { and } \Omega^{C}
$$

(c8) constitutive relations :

$$
\begin{array}{ll}
\mathbf{D}=\in_{0} \mathbf{E} & \text { in } \Omega^{A} \\
\left\{\begin{array}{l}
\boldsymbol{\sigma} \\
\mathbf{D}
\end{array}\right\}=\tilde{\mathbf{C}}\left\{\begin{array}{c}
\boldsymbol{\epsilon} \\
\mathbf{E}
\end{array}\right\}=\left[\begin{array}{cc}
\mathbf{C} & -\mathbf{e}^{T} \\
\mathbf{e} & \in
\end{array}\right]\left\{\begin{array}{c}
\boldsymbol{\epsilon} \\
\mathbf{E}
\end{array}\right\} & \text { in } \Omega^{B} \text { and } \Omega^{C}
\end{array}
$$

where $\epsilon_{0}$ is the permittivity of air and $\tilde{\mathbf{C}}$ are self-defined.

(c9) compatibility conditions :

$$
\left.\left(\mathbf{u}^{B}-\mathbf{u}^{C}\right)\right|_{\theta=\theta_{B C}}=\mathbf{0},\left.\left(\phi^{B}-\phi^{C}\right)\right|_{\theta=\theta_{B C}}=0,\left.\left(\phi^{C}-\phi^{A}\right)\right|_{\theta=\theta_{C A}}=0,\left.\left(\phi^{A}-\phi^{B}\right)\right|_{\theta=\theta_{A B}}=0
$$

in which $\mathbf{u}=\left\{u_{r}, u_{\theta}, u_{z}\right\}^{T}$. Moreover, the compatibility conditions imply the following conditions on the displacement variation $\delta \mathbf{u}$ and the electric potential variation $\delta \phi$ :

$$
\left.\left(\delta \mathbf{u}^{B}-\delta \mathbf{u}^{C}\right)\right|_{\theta=\theta_{B C}}=\mathbf{0},\left.\left(\delta \phi^{B}-\delta \phi^{C}\right)\right|_{\theta=\theta_{B C}}=0,\left.\left(\delta \phi^{C}-\delta \phi^{A}\right)\right|_{\theta=\theta_{C A}}=0,\left.\left(\delta \phi^{A}-\delta \phi^{B}\right)\right|_{\theta=\theta_{A B}}=0
$$

In the interfacial conditions (c3), (c4), (c5) and (c9), the field variables defined in $\Omega^{A}, \Omega^{B}$ and $\Omega^{C}$ are followed by superscripts " $A$ ", " $B$ " and " $C$ ", respectively. Moreover, $\theta_{C A}, \theta_{B C}, \theta_{A B}$ are the angular coordinates of the three subdomain interfaces as shown in Figure 1. Air and piezoelectrics are mechanically uncoupled.

\section{WEAK FORMULATION FOR PERMEABLE NOTCH IN PIEZOELECTRICS}

With conditions (c6) to (c8) taken as the subsidiary conditions which are satisfied as a priori, stress, strain, electric displacement and electric field are derived from displacement and electric potential. By further constraining (c9) or, equivalently, requiring the displacement and electric potential to be compatible, the following integral equation is the weak form of the remaining governing equations ( (c1) to (c5)) :

$$
\begin{aligned}
\int_{\Omega^{A}} \frac{1}{r} \delta & \phi \boldsymbol{\nabla}(r \mathbf{D}) d \Omega-\int_{\Omega^{B} \cup \Omega^{C}} \frac{1}{r}\left\{\begin{array}{c}
\delta \mathbf{u} \\
-\delta \phi
\end{array}\right\}^{T}\left\{\begin{array}{c}
\boldsymbol{\nabla}_{m}(r \boldsymbol{\sigma}) \\
\boldsymbol{\nabla}(r \mathbf{D})
\end{array}\right\} d \Omega+\left.\int_{0}^{R} \int_{0}^{L}\left(\left\{\begin{array}{c}
\delta \mathbf{u} \\
-\delta \phi
\end{array}\right\}^{T}\left\{\begin{array}{c}
\boldsymbol{\sigma}_{\theta}^{B}-\boldsymbol{\sigma}_{\theta}^{C} \\
D_{\theta}^{B}-D_{\theta}^{C}
\end{array}\right\}\right)\right|_{\theta=\theta_{B C}} d z d r \\
& +\left.\int_{0}^{R} \int_{0}^{L}\left(\delta \mathbf{u}^{T} \boldsymbol{\sigma}_{\theta}^{C}\right)\right|_{\theta=\theta_{C A}} d z d r-\left.\int_{0}^{R} \int_{0}^{L}\left(\delta \mathbf{u}^{T} \boldsymbol{\sigma}_{\theta}^{B}\right)\right|_{\theta=\theta_{A B}} d z d r
\end{aligned}
$$




$$
-\left.\int_{0}^{R} \int_{0}^{L}\left[\delta \phi\left(D_{\theta}^{C}-D_{\theta}^{A}\right)\right]\right|_{\theta=\theta_{C A}} d z d r-\left.\int_{0}^{R} \int_{0}^{L}\left[\delta \phi\left(D_{\theta}^{A}-D_{\theta}^{B}\right)\right]\right|_{\theta=\theta_{A B}} d z d r=0
$$

where $\delta$ is the variational symbol. It can be seen that $\delta \phi$ enforces (c2) and (c5) whereas $\delta \mathbf{u}$ enforces (c1), (c3) and (c4). By invoking the divergence theorem, see Appendix A, the above weak form can be expressed as:

$$
\begin{gathered}
\left.\int_{\Omega^{B} \cup \Omega^{C}}\left(\begin{array}{c}
\delta \boldsymbol{\epsilon} \\
\delta \mathbf{E}
\end{array}\right\}^{T}\left\{\begin{array}{c}
\mathbf{\sigma} \\
\mathbf{D}
\end{array}\right\}\right) d \Omega+\int_{\Omega^{A}}\left(\delta \mathbf{E}^{T} \mathbf{D}\right) d \Omega-\left.R \int_{\theta_{A B}}^{\theta_{C A}} \int_{0}^{L}\left(\left\{\begin{array}{c}
\delta \mathbf{u} \\
-\delta \phi
\end{array}\right\}^{T}\left\{\begin{array}{c}
\boldsymbol{\sigma}_{r} \\
D_{r}
\end{array}\right\}\right)\right|_{r=R} d z d \theta \\
+R \int_{\theta_{C A}}^{\theta_{A B}} \int_{0}^{L}\left(\delta \phi D_{r}\right) d \theta d z-\left.\int_{0}^{R} \int_{\theta_{A B}}^{\theta_{C A}}\left[r\left\{\begin{array}{c}
\delta \mathbf{u} \\
-\delta \phi
\end{array}\right\}^{T}\left\{\begin{array}{c}
\boldsymbol{\sigma}_{z} \\
D_{z}
\end{array}\right\}\right]\right|_{z=0} ^{z=L} d r d \theta+\left.\int_{0}^{R} \int_{\theta_{C A}}^{\theta_{A B}}\left(r \delta \phi D_{z}\right)\right|_{z=0} ^{z=L} d r d \theta=0
\end{gathered}
$$

It appears that a functional from which Eqn.(3) can be derived does not exist. With $\boldsymbol{\sigma}$ and D expressed in terms of $\mathbf{\epsilon}$ and $\mathbf{E}$ via (c8), it can be seen that the weak form is not symmetric with respect to $(\boldsymbol{\epsilon}, \mathbf{E})$ and $(\delta \boldsymbol{\epsilon}, \delta \mathbf{E})$. In other word, the weak form is not self-adjoint.

\section{F.E. FORMULATION FOR PERMEABLE NOTCH IN PIEZOELECTRICS}

The weak form in Eqn.(3) can be generalized to include more subdomains and, thus, is applicable to finite element discretization. Following the previous analytical works on eigen-solution analysis of piezoelectrics (e.g. Sosa \& Pak 1990; Kuo \& Barnett 1991; Qin \& Yu 1997), eigensolutions for the displacement and electric potential are assumed to possess the parametric forms below :

$$
\mathbf{u}=\left\{\begin{array}{l}
u_{r}(r, \theta) \\
u_{\theta}(r, \theta) \\
u_{z}(r, \theta)
\end{array}\right\}=r^{\lambda+1}\left\{\begin{array}{l}
U_{r}(\theta) \\
U_{\theta}(\theta) \\
U_{z}(\theta)
\end{array}\right\}=r^{\lambda+1} \mathbf{U}(\theta) \quad \text { and } \quad \phi=\phi(r, \theta)=r^{\lambda+1} \Phi(\theta)
$$

in which $\lambda$ is the eigenvalue. The eigenfunctions $U_{r}, U_{\theta}, U_{z}$ and $\Phi$ are zeroth order continuous and belong to the Sobolev space of degree one. The above eigensolutions are independent of the longitudinal or edge coordinate $z$ and, thus, can only accounts for edge singularities. All the displacement components and the electric potential are assumed to possess the same $r$-dependence so that the stress and electric displacement derived from them would possess the same order of singularity.

With Eqn.(4) invoked, the last two terms in the left hand side of Eqn.(3) vanish due to the $z$ independence of the field variables and the latter equation becomes: 


$$
\begin{aligned}
\left.\sum_{e^{\text {piezo }}} \int_{\Omega^{e}}\left(\begin{array}{c}
\delta \mathbf{\epsilon} \\
\delta \mathbf{E}
\end{array}\right\}^{T}\left\{\begin{array}{c}
\boldsymbol{\sigma} \\
\mathbf{D}
\end{array}\right\}\right) d \Omega & \left.-\sum_{e^{\text {piezo }}} R \int_{\theta_{S}^{e}}^{\theta_{T}^{e}} \int_{0}^{L}\left[\begin{array}{c}
\delta \mathbf{u} \\
-\delta \phi
\end{array}\right\}^{T}\left\{\begin{array}{c}
\boldsymbol{\sigma}_{r} \\
D_{r}
\end{array}\right\}\right]\left.\right|_{r=R} d z d \theta \\
& +\sum_{e^{a i r}} \int_{\Omega^{e}}\left(\delta \mathbf{E}^{T} \mathbf{D}\right) d \Omega+\sum_{e^{a i r}} R \int_{\theta_{S}^{e}}^{\theta_{T}^{e}} \int_{0}^{L} \delta \phi D_{r} d \theta d z=0
\end{aligned}
$$

in which " $e^{\text {piezo" }}$ and " $e^{\text {air } " ~ d e s i g n a t e ~ t h e ~ e l e m e n t s ~ m o d e l i n g ~ t h e ~ p i e z o e l e c t r i c s ~ a n d ~ a i r ~ r e s p e c t i v e l y . ~}$ Each of the elements is defined by $r \in[0, R], \theta \in\left[\theta_{S}^{e}, \theta_{T}^{e}\right]$ and $z \in[0, L]$. The angular coordinate $\theta$ and natural coordinate $\xi \in[-1,+1]$ are related as :

$$
\theta=N_{S} \theta_{S}^{e}+N_{T} \theta_{T}^{e}
$$

where $N_{S}=(1-\xi) / 2$ and $N_{T}=(1+\xi) / 2$. Hence,

$$
J=\frac{d \theta}{d \xi}=\frac{1}{2}\left(\theta_{T}^{e}-\theta_{S}^{e}\right), \frac{d}{d \theta}=\frac{1}{J} \frac{d}{d \xi}=\frac{2}{\theta_{T}^{e}-\theta_{S}^{e}} \frac{d}{d \xi}
$$

It should be remarked that the displacement and electric potential continuity at the interfaces of different materials should be maintained at $\mathrm{C}^{0}$. Any higher order continuity would lead to continuous strain and electric field. Consequently, the traction and charge reciprocity conditions at the interfaces of different media can never be satisfied. With respect to the parametric assumption in Eqn.(4), the element displacement and electric potential can be assumed as:

$$
\begin{gathered}
\mathbf{u}=r^{\lambda+1}\left[N_{S} \mathbf{I}_{3}, N_{1} \mathbf{I}_{3}, \ldots, N_{n} \mathbf{I}_{3}, N_{T} \mathbf{I}_{3}\right]\left\{\begin{array}{c}
\mathbf{U}_{S}^{e} \\
\vdots \\
\mathbf{U}_{T}^{e}
\end{array}\right\}=r^{\lambda+1}\left[N_{S} \mathbf{I}_{3}, N_{1} \mathbf{I}_{3}, \ldots, N_{n} \mathbf{I}_{3}, N_{T} \mathbf{I}_{3}\right] \mathbf{q}_{m}^{e} \\
\phi=r^{\lambda+1}\left[N_{S}, N_{1}, \ldots, N_{n}, N_{T}\right]\left\{\begin{array}{c}
\Phi_{S}^{e} \\
\vdots \\
\Phi_{T}^{e}
\end{array}\right\}=-r^{\lambda+1}\left[-N_{S},-N_{1}, \ldots,-N_{n},-N_{T}\right] \mathbf{q}_{e}^{e}=-r^{\lambda+1} \mathbf{N} \mathbf{q}_{e}^{e}
\end{gathered}
$$

Thus,

$$
\left\{\begin{array}{c}
\mathbf{u} \\
-\phi
\end{array}\right\}=r^{\lambda+1}\left[\begin{array}{cc}
{\left[N_{S} \mathbf{I}_{3}, N_{1} \mathbf{I}_{3}, \ldots, N_{n} \mathbf{I}_{3}, N_{T} \mathbf{I}_{3}\right]} & \mathbf{0}_{3 \times(2+n)} \\
\mathbf{0}_{1 \times(6+3 n)} & {\left[-N_{S},-N_{1}, \ldots,-N_{n},-N_{T}\right]}
\end{array}\right]\left\{\begin{array}{l}
\mathbf{q}_{m}^{e} \\
\mathbf{q}_{e}^{e}
\end{array}\right\}=r^{\lambda+1} \widetilde{\mathbf{N}}\left\{\begin{array}{c}
\mathbf{q}_{m}^{e} \\
\mathbf{q}_{e}^{e}
\end{array}\right\}
$$

where $\mathbf{I}_{3}$ is the third order identity matrix, $N_{i}=\xi^{i-1}\left(1-\xi^{2}\right)$ is the bubble shape function, $n$ is the number of bubble modes, $\mathbf{U}_{S}^{e}$ and $\mathbf{U}_{T}^{e}$ are respectively the generalized nodal displacement vectors at $\theta_{S}^{e}$ and $\theta_{T}^{e} ; \Phi_{S}^{e}$ and $\Phi_{T}^{e}$ are respectively the generalized nodal electrical potentials at $\theta_{S}^{e}$ and $\theta_{T}^{e}$; $\mathbf{q}_{m}^{e}$ and $\mathbf{q}_{e}^{e}$ are respectively the element vectors of generalized displacement (mechanical) and 
electrical potential (electric) d.o.f.s.

Starting from Eqn.(10), the remaining field variables in an element modeling piezoelectrics can be derived by invoking conditions (c6), (c7) and (c8) in Section 3 as :

$$
\begin{aligned}
& \left\{\begin{array}{c}
\mathbf{\epsilon} \\
\mathbf{E}
\end{array}\right\}=r^{\lambda}\left(\lambda \tilde{\mathbf{B}}_{1}+\tilde{\mathbf{B}}_{0}\right)\left\{\begin{array}{l}
\mathbf{q}_{m}^{e} \\
\mathbf{q}_{e}^{e}
\end{array}\right\},\left\{\begin{array}{l}
\boldsymbol{\sigma} \\
\mathbf{D}
\end{array}\right\}=\tilde{\mathbf{C}}\left\{\begin{array}{c}
\mathbf{\epsilon} \\
\mathbf{E}
\end{array}\right\}=r^{\lambda}\left(\lambda \tilde{\mathbf{C}} \tilde{\mathbf{B}}_{1}+\tilde{\mathbf{C}} \tilde{\mathbf{B}}_{0}\right)\left\{\begin{array}{l}
\mathbf{q}_{m}^{e} \\
\mathbf{q}_{e}^{e}
\end{array}\right\}, \\
& \left\{\begin{array}{c}
\boldsymbol{\sigma}_{r} \\
D_{r}
\end{array}\right\}=r^{\lambda} \tilde{\mathbf{H}}\left(\lambda \tilde{\mathbf{B}}_{1}+\tilde{\mathbf{B}}_{0}\right)\left\{\begin{array}{l}
\mathbf{q}_{m}^{e} \\
\mathbf{q}_{e}^{e}
\end{array}\right\}
\end{aligned}
$$

where

$$
\begin{aligned}
& \widetilde{\mathbf{B}}_{1}=\left[\begin{array}{ccccc:cccc}
\widetilde{\mathbf{B}}_{1 m S} & \widetilde{\mathbf{B}}_{1 m 1} & \cdots & \widetilde{\mathbf{B}}_{1 m n} & \widetilde{\mathbf{B}}_{1 m T} & \multicolumn{4}{|c}{} \\
\hdashline & \mathbf{0}_{3 \times(3 n+6)} & & \widetilde{\mathbf{B}}_{1 e s} & \widetilde{\mathbf{B}}_{1 e 1} & \cdots & \widetilde{\mathbf{B}}_{1 e n} & \widetilde{\mathbf{B}}_{1 e T}
\end{array}\right],
\end{aligned}
$$

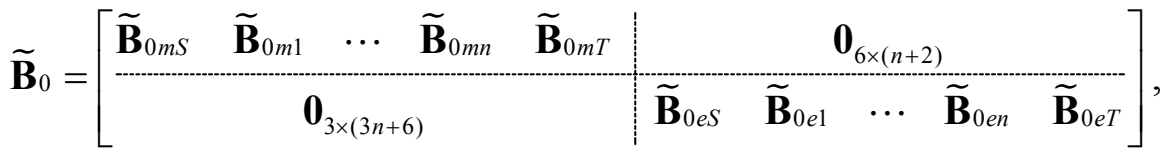

$$
\begin{aligned}
& \tilde{\mathbf{H}}=\left[\begin{array}{lllllllll}
1 & 0 & 0 & 0 & 0 & 0 & 0 & 0 & 0 \\
0 & 0 & 0 & 0 & 0 & 1 & 0 & 0 & 0 \\
0 & 0 & 0 & 0 & 1 & 0 & 0 & 0 & 0 \\
0 & 0 & 0 & 0 & 0 & 0 & 1 & 0 & 0
\end{array}\right] \tilde{\mathbf{C}} .
\end{aligned}
$$

In matrices $\widetilde{\mathbf{B}}_{0}$ and $\widetilde{\mathbf{B}}_{1}$,

$$
\widetilde{\mathbf{B}}_{1 m j}=\left[\begin{array}{ccc}
N_{j} & 0 & 0 \\
0 & 0 & 0 \\
0 & 0 & 0 \\
0 & 0 & 0 \\
0 & 0 & N_{j} \\
0 & N_{j} & 0
\end{array}\right], \widetilde{\mathbf{B}}_{0 m}=\left[\begin{array}{ccc}
N_{j} & 0 & 0 \\
N_{j} & N_{j}, \theta & 0 \\
0 & 0 & 0 \\
0 & 0 & N_{j}, \theta_{\theta} \\
0 & 0 & N_{j} \\
N_{j}, \theta & 0 & 0
\end{array}\right], \widetilde{\mathbf{B}}_{0 e}=\left[\begin{array}{c}
-N_{j} \\
-N_{j, \theta} \\
0
\end{array}\right], \widetilde{\mathbf{B}}_{1 e j}=\left[\begin{array}{c}
-N_{j} \\
0 \\
0
\end{array}\right]
$$

in which $j=S, 1, \ldots, n, T$ and $N_{j},_{\theta}=d N_{j} / d \theta$. On the other hand, the electric field and electric displacement in an element modeling air can be derived from Eqn.(9) by invoking conditions (c7) and (c8) in Section 2 as :

$$
\mathbf{E}=r^{\lambda}\left(\lambda \mathbf{B}_{1}+\mathbf{B}_{0}\right) \mathbf{q}_{e}^{e}, \mathbf{D}=\epsilon_{o} \mathbf{E}=r^{\lambda} \in_{o}\left(\lambda \mathbf{B}_{1}+\mathbf{B}_{0}\right) \mathbf{q}_{e}^{e}, D_{r}=r^{\lambda} \in_{o} \mathbf{H}\left(\lambda \mathbf{B}_{1}+\mathbf{B}_{0}\right) \mathbf{q}_{e}^{e}
$$

in which

$$
\mathbf{H}=\left[\begin{array}{lll}
1 & 0 & 0
\end{array}\right], \mathbf{B}_{1}=-\left[\begin{array}{ccccc}
N_{S} & N_{1} & \ldots & N_{n} & N_{T} \\
0 & 0 & \ldots & 0 & 0 \\
0 & 0 & \ldots & 0 & 0
\end{array}\right], \mathbf{B}_{0}=-\left[\begin{array}{ccccc}
N_{S} & N_{1} & \ldots & N_{n} & N_{T} \\
N_{S},{ }_{\theta} & N_{1},{ }_{\theta} & \ldots & N_{n}, \theta & N_{T},{ }_{\theta} \\
0 & 0 & \ldots & 0 & 0
\end{array}\right] .
$$

By substituting Eqn.(9) to Eqn.(13) into Eqn.(5), the latter becomes : 


$$
\sum_{e^{p i e z o}}\left[\left\{\begin{array}{l}
\delta \mathbf{q}_{m}^{e} \\
\delta \mathbf{q}_{e}^{e}
\end{array}\right\}^{T}\left(\lambda^{2} \widetilde{\mathbf{P}}^{e}+\lambda \widetilde{\mathbf{Q}}^{e}+\widetilde{\mathbf{R}}^{e}\right)\left\{\begin{array}{l}
\mathbf{q}_{m}^{e} \\
\mathbf{q}_{e}^{e}
\end{array}\right\}\right]+\sum_{e^{a i r}}\left[\left(\delta \mathbf{q}_{e}^{e}\right)^{T}\left(\lambda^{2} \mathbf{P}^{e}+\lambda \mathbf{Q}^{e}+\mathbf{R}^{e}\right) \mathbf{q}_{e}^{e}\right]=0
$$

where

$$
\begin{aligned}
& \widetilde{\mathbf{P}}^{e}=\int_{\theta_{S}^{e}}^{\theta_{T}^{e}}\left(\widetilde{\mathbf{B}}_{1}^{T} \widetilde{\mathbf{C}} \widetilde{\mathbf{B}}_{1}-2 \widetilde{\mathbf{N}}^{T} \widetilde{\mathbf{H}} \widetilde{\mathbf{B}}_{1}\right) d \theta, \widetilde{\mathbf{Q}}^{e}=\int_{\theta_{S}^{e}}^{\theta_{T}^{e}}\left[\widetilde{\mathbf{B}}_{1}^{T} \widetilde{\mathbf{C}}^{\mathbf{B}_{0}}+\widetilde{\mathbf{B}}_{0}^{T} \widetilde{\mathbf{C}}^{\mathbf{B}_{1}}-2 \widetilde{\mathbf{N}}^{T} \widetilde{\mathbf{H}}\left(\widetilde{\mathbf{B}}_{0}+\widetilde{\mathbf{B}}_{1}\right)\right] d \theta \\
& \widetilde{\mathbf{R}}^{e}=\int_{\theta_{S}^{e}}^{\theta_{T}^{e}}\left(\widetilde{\mathbf{B}}_{0}^{T} \widetilde{\mathbf{C}} \widetilde{\mathbf{B}}_{0}-2 \widetilde{\mathbf{N}}^{T} \widetilde{\mathbf{H}} \widetilde{\mathbf{B}}_{0}\right] d \theta, \mathbf{P}^{e}=\epsilon_{o} \int_{\theta_{S}^{e}}^{\theta_{T}^{e}}\left(\mathbf{B}_{1}^{T} \mathbf{B}_{1}-2 \mathbf{N}^{T} \mathbf{H} \mathbf{B}_{1}\right) d \theta \\
& \mathbf{Q}^{e}=\epsilon_{o} \int_{\theta_{T}^{e}}^{\theta_{T}^{e}}\left[\mathbf{B}_{1}^{T} \mathbf{B}_{0}+\mathbf{B}_{0}^{T} \mathbf{B}_{1}-2 \mathbf{N}^{T} \mathbf{H}\left(\mathbf{B}_{0}+\mathbf{B}_{1}\right)\right] d \theta, \mathbf{R}^{e}=\epsilon_{o} \int_{\theta_{S}^{e}}^{\theta_{T}^{e}}\left(\mathbf{B}_{0}^{T} \mathbf{B}_{0}-2 \mathbf{N}^{T} \mathbf{H} \mathbf{B}_{0}\right) d \theta
\end{aligned}
$$

As $\delta \mathbf{q}_{m}^{e}$ 's and $\delta \mathbf{q}_{e}^{e}$ 's are arbitrary, the following second order characteristic matrix equation can be obtained :

$$
\left(\lambda^{2} \mathbf{P}+\lambda \mathbf{Q}+\mathbf{R}\right) \mathbf{q}=\mathbf{0}
$$

in which $\mathbf{q}$ is the collection of all $\mathbf{q}_{m}^{e}$ 's and $\mathbf{q}_{e}^{e}$ 's, $\mathbf{P}$ is assembled from $\widetilde{\mathbf{P}}^{e}$ and $\mathbf{P}^{e}, \mathbf{Q}$ is assembled from $\widetilde{\mathbf{Q}}^{e}$ and $\mathbf{Q}^{e}, \mathbf{R}$ is assembled from $\widetilde{\mathbf{R}}^{e}$ and $\mathbf{R}^{e}$. As the weak form is not self-adjoint, the matrices are not symmetric. The above equation can be transformed into the standard first order eigensolution problem by introducing a dummy vector $\tilde{\mathbf{q}}=\lambda \mathbf{q}$ :

$$
\left[\begin{array}{cc}
-\mathbf{P}^{-1} \mathbf{Q} & -\mathbf{P}^{-1} \mathbf{R} \\
\mathbf{I} & \mathbf{0}
\end{array}\right]\left\{\begin{array}{l}
\tilde{\mathbf{q}} \\
\mathbf{q}
\end{array}\right\}=\lambda\left\{\begin{array}{c}
\tilde{\mathbf{q}} \\
\mathbf{q}
\end{array}\right\}
$$

where $\mathbf{I}$ is the identity matrix with order equal to $\operatorname{dim} .(\mathbf{q})$. As the matrix in the left hand side of the equation is not symmetric, the eigensolutions need not be real.

\section{F.E. FORMULATION FOR IMPERMEABLE NOTCH IN PIEZOELECTRICS}

The permittivity for most piezoelectrics are larger than that of air $\epsilon_{o}$ by more than one order of magnitude. By neglecting $\epsilon_{o}$, electric displacement inside the air would be zero. The last two charge-reciprocity conditions in (c5) of Section 2 become the charge-free conditions, i.e. $\left.D_{\theta}^{C}\right|_{\theta=\theta_{C A}}=\left.D_{\theta}^{B}\right|_{\theta=\theta_{A B}}=0$. On the other hand, the last two compatibility conditions in (c9) of Section 2 can be discarded. As the air and piezoelectrics are uncoupled, the former does not need to be modelled. The weak form and the finite element equation of the present eigensolution problem can be obtained by setting $\mathbf{D}$ in $\Omega^{A}$ and $\epsilon_{o}$ to zero respectively in Eqn.(3) and Eqn.(14), i.e. 


$$
\begin{array}{r}
\left.\int_{\Omega^{B} \cup \Omega^{C}}\left\{\begin{array}{c}
\delta \boldsymbol{\epsilon} \\
\delta E
\end{array}\right\}^{T}\left\{\begin{array}{c}
\boldsymbol{\sigma} \\
D
\end{array}\right\}\right) d \Omega-\left.R \int_{\theta_{A B}}^{\theta_{C A}} \int_{z_{S}}^{z_{T}}\left(\left\{\begin{array}{c}
\delta \mathbf{u} \\
-\delta \phi
\end{array}\right\}^{T}\left\{\begin{array}{c}
\boldsymbol{\sigma}_{r} \\
D_{r}
\end{array}\right\}\right)\right|_{r=R} d z d \theta \\
-\left.\int_{0}^{R} \int_{\theta_{A B}}^{\theta_{C A}}\left[r\left\{\begin{array}{c}
\delta \mathbf{u} \\
-\delta \phi
\end{array}\right\}^{T}\left\{\begin{array}{c}
\boldsymbol{\sigma}_{z} \\
D_{z}
\end{array}\right\}\right]\right|_{z=0} ^{z=L} d r d \theta=0
\end{array}
$$

and

$$
\sum_{e^{\text {piezo }}}\left\{\begin{array}{l}
\delta \mathbf{q}_{m}^{e} \\
\delta \mathbf{q}_{e}^{e}
\end{array}\right\}^{T}\left(\lambda^{2} \widetilde{\mathbf{P}}^{e}+\lambda \widetilde{\mathbf{Q}}^{e}+\widetilde{\mathbf{R}}^{e}\right)\left\{\begin{array}{l}
\mathbf{q}_{m}^{e} \\
\mathbf{q}_{e}^{e}
\end{array}\right\}=0
$$

where matrices have been defined in Eqn.(14). A second order characteristic equation of the same form as Eqn.(15) can be formed after assembling the matrices in Eqn.(18).

\section{F.E. FORMULATION FOR CONDUCTING CRACK IN PIEZOELECTRICS}

It is argued that the air gap or the crack can be practically conducting when the crack angle is sufficiently small even for the low permittivity of air. Thus, the crack faces will be equipotential and air does not need to be modeled electrically. The condition considered here is another case of uncoupled air and piezoelectrics. The characteristic equation formed for impermeable notch can equally applied to the present case except that the one of the two nodal generalized electric potentials at $\theta_{C A}$ and $\theta_{A B}$ have to be condensed from equation by the following equal-potential constraint :

$$
\left.\Phi^{B}\right|_{\theta=\theta_{A B}}=\left.\Phi^{C}\right|_{\theta=\theta_{C A}}
$$

\section{NUMERICAL EXAMPLES}

A number of examples will be studied to demonstrate the efficacy of the devised finite element procedures. Only the eigenvalues whose real parts are within minus unity and zero, i.e. $\operatorname{Re}(\lambda) \in[-$ $1,0]$, will be reported. For $\operatorname{Re}(\lambda) \geq 0$, the stress and electric displacement are not singular. $\operatorname{For} \operatorname{Re}(\lambda)$ $<-1$, the electromechanical energy stored in the material is unbounded and the corresponding eigensolutions are rejected on physical ground. Unless specified, the number of bubble modes in all elements are set to seven with which the eigenvalues have converged at least to the sixth significant figures. All the computations are conducted in double precision that employs sixteen significant figures.

Tables $1 \mathrm{a}$ and $1 \mathrm{~b}$ list the non-zero stiffness, piezoelectric and permittivity coefficients of the 
media to be considered (see Kuo \& Barnett 1991; Park \& Sun 1995) with respect to their principal material directions 1,2 and 3 among which 3 is the poling direction. Besides barium sodium niobate, the other two piezoelectric media are transverse isotropic. Taking PZT-4 and air as examples whose constitutive relations can be denoted as :

$$
\left\{\begin{array}{c}
\boldsymbol{\sigma}\left[\frac{N}{m^{2}}\right] \\
\mathbf{D}\left[\frac{C}{m^{2}}\right]
\end{array}\right\}=\left[\begin{array}{cc}
\sim 10^{11}\left[\frac{N}{m^{2}}\right] & \sim 10^{1}\left[\frac{C}{m^{2}}\right] \\
\sim 10^{1}\left[\frac{C}{m^{2}}\right] & \sim 10^{-9}\left[\frac{C}{V m}=\frac{C^{2}}{N m^{2}}\right]
\end{array}\right]\left\{\begin{array}{c}
\boldsymbol{\epsilon} \\
\mathbf{E}\left[\frac{V}{m}=\frac{N}{C}\right]
\end{array}\right\} \quad \text { for PZT-4 }
$$

and

$$
\mathbf{D}\left[\frac{C}{m^{2}}\right]=\sim 10^{-12}\left[\frac{C}{V m}=\frac{C^{2}}{N m^{2}}\right] \cdot \mathbf{E}\left[\frac{V}{m}=\frac{N}{C}\right] \quad \text { for air }
$$

Table 1a. Non-zero stiffness coefficients (in $\mathrm{GN} / \mathrm{m}^{2}$ ), $C_{i j}=C_{i i}$

\begin{tabular}{|c|ccccccccc|}
\hline Medium & $\mathrm{C}_{11}$ & $\mathrm{C}_{22}$ & $\mathrm{C}_{33}$ & $\mathrm{C}_{44}$ & $\mathrm{C}_{55}$ & $\mathrm{C}_{66}$ & $\mathrm{C}_{12}$ & $\mathrm{C}_{13}$ & $\mathrm{C}_{23}$ \\
\hline PZT-4 & 139 & 139 & 113 & 25.6 & 25.6 & 30.6 & 77.8 & 74.3 & 74.3 \\
cadmium sulfide & 90.7 & 90.7 & 93.8 & 15.04 & 15.04 & 16.3 & 58.1 & 51 & 51 \\
barium sodium niobate & 239 & 247 & 135 & 66 & 66 & 76 & 104 & 50 & 52 \\
\hline
\end{tabular}

Table 1b. Non-zero piezoelectric coefficients (in $\mathrm{C} / \mathrm{m}^{2}$ ) and permittivity coefficients (in $\mathrm{pC} / \mathrm{Vm}$ )

\begin{tabular}{|c|ccccc|ccc|}
\hline Medium & $e_{15}$ & $e_{24}$ & $e_{31}$ & $e_{32}$ & $e_{33}$ & $\epsilon_{11}$ & $\epsilon_{22}$ & $\epsilon_{33}$ \\
\hline PZT-4 & 13.44 & 13.44 & -6.98 & -6.98 & 13.84 & 6000 & 6000 & 5470 \\
cadmium sulfide & -0.21 & -0.21 & -0.24 & -0.24 & 0.44 & 79.86 & 79.86 & 84.38 \\
barium sodium niobate & 2.8 & 3.4 & -0.4 & -0.3 & 4.3 & 1966 & 2010 & 283.3 \\
air & --- & --- & --- & --- & --- & 8.854 & 8.854 & 8.854 \\
\hline
\end{tabular}

With the bracketed standard units for the constitutive relation, the IMSL ${ }^{T M}$ FORTRAN subroutine DEVCRG declines to compute any eigen-solution due to the ill-conditioning of the system matrices. To resolve the problem, the set of units employed in the following relations are employed :

$$
\left\{\begin{array}{c}
\boldsymbol{\sigma}\left[\frac{\mathrm{N}}{\mathrm{mm}^{2}}\right] \\
\mathbf{D}\left[\frac{\mathrm{pC}}{\mathrm{mm}^{2}}\right]
\end{array}\right\}=\left[\begin{array}{cc}
\sim 10^{5}\left[\frac{\mathrm{N}}{\mathrm{mm}^{2}}\right] & \sim 10^{7}\left[\frac{\mathrm{pC}}{\mathrm{mm}^{2}}\right] \\
\sim 10^{7}\left[\frac{\mathrm{pC}}{\mathrm{mm}^{2}}\right] & \sim 10^{9}\left[\frac{(\mathrm{pC})^{2}}{N(\mathrm{~mm})^{2}}\right]
\end{array}\right]\left\{\begin{array}{c}
\boldsymbol{\epsilon} \\
\mathbf{E}\left[\frac{\mathrm{N}}{p C}\right]
\end{array}\right\} \quad \text { for PZT-4 }
$$

and

$$
\mathbf{D}\left[\frac{p C}{m m^{2}}\right]=\sim 10^{6}\left[\frac{(p C)^{2}}{N(m m)^{2}}\right] \cdot \mathbf{E}\left[\frac{N}{p C}\right]
$$

for air

Alternatively, preconditioning techniques can be applied to the characteristic matrix equation to yield better conditioned system matrices. 


\subsection{Impermeable and Conducting Cracks in Homogeneous Piezoelectrics}

The homogeneous piezoelectrics is modeled by two equal-sized elements as shown in Figure 2a. The poling direction is aligned with z-axis. The numbers of bubble modes $n_{A}$ and $n_{B}$ insides the two elements are varied and the results are listed in Table 2 and Table 3 for impermeable and conducting cracks in PZT-4 and calcium sulfide, respectively. In all eigensolutions, the analytical eigenvlaue $\lambda$ 's are $-1 / 2$. The computed results converge to their analytical counterparts as the number of bubble modes is increased.

It also noted in the computed results that the eigensolutions for mode I, II and III are not affected by the considered electric boundary conditions. Moreover, only the radial and circumferential displacements are non-zero for mode I and mode II. On the other hand, only the longitudinal displacement is non-zero for mode III whereas mode IV is featured by zero displacements but nonzero electric potential. (Parton 1976, Mcmeeking 1989, Pak 1990, Sosa \& Pak 1990, Sosa 1992, Suo, Kuo, Barnett \& Willis 1992). Hence, modes I to III are mechanical fracture modes whereas mode IV is electric fracture mode. Under the conducting condition, mode IV does not exist. All these observations are in compliance with the analytical implications discussed in Appendix B.

In most, if not all, research works on eigensolution problems of conventional and piezoelectric materials, analytical solutions for the eigenfunctions $\mathbf{U}(\theta)$ and $\Phi(\theta)$ are not reported. To illustrate that the present procedure can equally yield accurate eigenfunctions, the case of impermeable crack is considered. It can be deduced from the discussion in Appendix B that eigenfunctions for modes I, II, III and IV are identical to that of modes I, II, III and III for plain strain crack in isotropic materials (see, e.g., Anderson 1995) :

$\begin{array}{ll}\text { Mode I : } & \left\{\begin{array}{l}U_{r} \\ U_{\theta}\end{array}\right\}=\left[\begin{array}{cc}\cos \theta & \sin \theta \\ -\sin \theta & \cos \theta\end{array}\right]\left\{\begin{array}{l}{\left[1-2 v+\sin ^{2}(\theta / 2)\right] \cos (\theta / 2)} \\ {\left[2-2 v+\cos ^{2}(\theta / 2)\right] \sin (\theta / 2)}\end{array}\right\}, \\ \text { Mode II : } & \left\{\begin{array}{l}U_{r} \\ U_{\theta}\end{array}\right\}=\left[\begin{array}{cc}\cos \theta & \sin \theta \\ -\sin \theta & \cos \theta\end{array}\right]\left\{\begin{array}{c}{\left[2-2 v+\cos ^{2}(\theta / 2)\right] \sin (\theta / 2)} \\ {\left[-1+2 v+\sin ^{2}(\theta / 2)\right] \cos (\theta / 2)}\end{array}\right\},\end{array}$

Mode III : $U_{z}=\sin (\theta / 2)$,

Mode IV : $\Phi=\sin (\theta / 2)$

where $v /(1-v)=C_{12} / C_{11}$. The above theoretical solutions and the present finite element predictions are plotted against $\theta$ in Figures 3, 4 and 5. Exceptionally closeness can be noted.

CPU time consumption has also been studied. A desktop computer with $450 \mathrm{MHz}$ Pentium CPU and Digital ${ }^{T M}$ Visual Fortran Version 6.0a is employed. Using the tenth and twentieth order Gaussian rules, the consumed CPU times are less than 0.98 and 1.12 seconds respectively for all the 
cases in Tables 2 and 3. The eigenvalues yielded by the two integration schemes agree with each other up to the seventh significant figure.

Table 2. Eigenvalues for impermeable and conducting cracks in PZT-4 poled along z-axis

\begin{tabular}{|c|c|c|c|c|}
\hline No. of d.o.f.s & electric b.c. & modes I \& II & mode III & mode IV \\
\hline $28\left(n_{A}=n_{B}=2\right)$ & impermeable & $-0.474769,-0.409591$ & -0.499966 & -0.499966 \\
$27\left(n_{A}=n_{B}=2\right)$ & conducting & ditto & ditto & nil \\
\hline $36\left(n_{A}=n_{B}=3\right)$ & impermeable & $-0.496870,-0.490333$ & -0.500000 & -0.500000 \\
$35\left(n_{A}=n_{B}=3\right)$ & conducting & ditto & ditto & nil \\
\hline $44\left(n_{A}=n_{B}=4\right)$ & impermeable & $-0.499785,-0.499350$ & -0.500000 & -0.500000 \\
$43\left(n_{A}=n_{B}=4\right)$ & conducting & ditto & ditto & nil \\
\hline $52\left(n_{A}=n_{B}=5\right)$ & impermeable & $-0.499990,-0.499972$ & -0.500000 & -0.500000 \\
$51\left(n_{A}=n_{B}=5\right)$ & conducting & ditto & ditto & nil \\
\hline $60\left(n_{A}=n_{B}=6\right)$ & impermeable & $-0.500000,-0.499999$ & -0.500000 & -0.500000 \\
$59\left(n_{A}=n_{B}=6\right)$ & conducting & ditto & ditto & nil \\
\hline $68\left(n_{A}=n_{B}=7\right)$ & impermeable & $-0.500000,-0.500000$ & -0.500000 & -0.500000 \\
$67\left(n_{A}=n_{B}=7\right)$ & conducting & ditto & ditto & nil \\
\hline
\end{tabular}

Table 3. Eigenvalues for impermeable and conducting slit-cracks in calcium sulfide poled along zaxis

\begin{tabular}{|c|c|c|c|c|}
\hline No. of nodal d.o.f.s & Crack face condition & modes I \& II & mode III & mode IV \\
\hline $28\left(n_{A}=n_{B}=2\right)$ & impermeable & $-0.473382,-0.405528$ & -0.499966 & -0.499966 \\
$27\left(n_{A}=n_{B}=2\right)$ & conducting & ditto & ditto & nil \\
\hline $36\left(n_{A}=n_{B}=3\right)$ & impermeable & $-0.496682,-0.489726$ & -0.500000 & -0.500000 \\
$35\left(n_{A}=n_{B}=3\right)$ & conducting & ditto & ditto & nil \\
\hline $44\left(n_{A}=n_{B}=4\right)$ & impermeable & $-0.499770,-0.499303$ & -0.500000 & -0.500000 \\
$43\left(n_{A}=n_{B}=4\right)$ & conducting & ditto & ditto & nil \\
\hline $52\left(n_{A}=n_{B}=5\right)$ & impermeable & $-0.499990,-0.499969$ & -0.500000 & -0.500000 \\
$51\left(n_{A}=n_{B}=5\right)$ & conducting & ditto & ditto & nil \\
\hline $60\left(n_{A}=n_{B}=6\right)$ & impermeable & $-0.500000,-0.499999$ & -0.500000 & -0.500000 \\
$59\left(n_{A}=n_{B}=6\right)$ & conducting & ditto & ditto & nil \\
\hline $68\left(n_{A}=n_{B}=7\right)$ & impermeable & $-0.500000,-0.500000$ & -0.500000 & -0.500000 \\
$67\left(n_{A}=n_{B}=7\right)$ & conducting & ditto & ditto & nil \\
\hline
\end{tabular}

\subsection{Impermeable Interfacial Cracks}

Following the example of Kuo \& Barnett (1991), impermeable interfacial cracks between dissimilar piezoelectrics are considered. For each of the materials, only one element is employed as 
shown in Figure 2a. To compare the present results directly with that of Kuo \& Barnett, the displacement and electric potential are taken to be proportional to $r^{\lambda}$ in and only in the present example (see Eqn.(4)). Hence, the real parts of the $\lambda$ 's associated with the physical singular fields would be bounded by zero and unity. Besides using different combinations of materials, the principal material directions ( 3 being the poling direction) are also varied and their direction cosines with respect to $\mathrm{x}-, \mathrm{y}$ - and z-coordinates are respectively given as first, second and third rows of matrices in Table 4. Kuo \& Barnett's results were obtained by forming a system of nonlinear equations using Stroh's formalism and the notion of surface impedance tensor. The equation system is then solved numerically by a LINPACK subroutine. It can be seen that the two sets of eigenvalues given by the present finite element method and Kuo \& Barnett (1991) are in excellent agreement.

Table 4. Eigenvalues for impermeable interfacial cracks

\begin{tabular}{|c|c|c|c|c|}
\hline $\begin{array}{c}\text { material in } \Omega^{B} \\
\text { (principal matl. directions) }\end{array}$ & \multicolumn{2}{|c|}{$\begin{array}{c}\text { material in } \Omega^{B} \\
\text { (principal matl. directions) }\end{array}$} & present & Kuo \& Barnett (1991) \\
\hline cadmium sulfide, & \multicolumn{2}{|c|}{ cadmium sulfide, } & & \\
\hline$\left(\begin{array}{lll}1 & 0 & 0\end{array}\right)$ & $\left(\begin{array}{ll}1 & 0\end{array}\right.$ & 0 & $\begin{array}{l}0.500000 \\
0.500000\end{array}$ & $\begin{array}{l}0.500000 \\
0.500000\end{array}$ \\
\hline $\begin{array}{lll}0 & 1 & 0\end{array}$ & $\begin{array}{ll}0 & 0.9239\end{array}$ & 0.3827 & 0.497815, & 0.497815, \\
\hline$\left(\begin{array}{lll}0 & 0 & 1\end{array}\right)$ & $0 \quad-0.3827$ & 0.9239 & 0.502186 & 0.502185 \\
\hline cadmium sulfide, & \multicolumn{2}{|c|}{ cadmium sulfide, } & & \\
\hline$\left(\begin{array}{lll}1 & 0 & 0\end{array}\right)$ & $\left(\begin{array}{ll}1 & 0\end{array}\right.$ & 0 & $\pm i 0.003547$ & $\pm i 0.003550$ \\
\hline 1 & $\begin{array}{ll}0 & 0.7071\end{array}$ & 0.7071 & 0.500000 & 0.500000 \\
\hline$\left(\begin{array}{ll}0 & 0\end{array}\right.$ & $\begin{array}{ll}0 & -0.7071\end{array}$ & 0.7071 & 0.500002 & 0.500000 \\
\hline cadmium sulfide, & \multicolumn{2}{|c|}{ barium sodium niobate, } & & \\
\hline$\left(\begin{array}{lll}1 & 0 & 0\end{array}\right)$ & $\left(\begin{array}{ll}1 & 0\end{array}\right.$ & 0 & $\pm i 0.024560$ & $\pm i 0.024555$ \\
\hline $0 \quad 1$ & 0.9239 & 0.3827 & 0.500000 & 0.500000 \\
\hline$\left(\begin{array}{lll}0 & 0 & 1\end{array}\right)$ & -0.3827 & 0.9239 & 0.500000 & 0.500000 \\
\hline cadmium sulfide & \multicolumn{2}{|c|}{ barium sodium niobate, } & 0.500002 & 0.500000 \\
\hline$\left(\begin{array}{lll}1 & 0 & 0\end{array}\right)$ & $0.2962 \quad 0.1710$ & 0.9397 & $\pm i 0.015755$ & $\pm i 0.015750$ \\
\hline 1 & -0.5000 & 0.0000 & 0.499725 & 0.499720 \\
\hline $0 \quad 0$ & -0.8138 & 0.3420 & 0.500289 & 0.500280 \\
\hline
\end{tabular}

\subsection{Permeable and Impermeable Notches in Homogeneous Piezoelectrics}

The homogeneous piezoelectrics is modeled by two equal-sized elements whereas air gap is also modeled by two equal-sized elements as shown in Figure 2b. Only small but finite notch angles are considered. The configurations mimics the cases of non-slit cracks (for cracks or slit crack, the notch angles are taken to be zero) or cracks opened by electromechanical loading. To our best knowledge, the eigensolution problem of permeable notches or cracks in which the air permittivity 
is taken into account has not been studied. Highly converged results are obtained by using nine and two bubble modes in the piezoelectric and air elements, if any, respectively. As the conducting boundary condition is no more realistic for finite notch anlges, it will be excluded in this subsection.

With $z$ taken to be the poling direction, Tables 5 and 6 list the computed eigenvalues for PZT-4 and cadmium sulfide, respectively. Same as Section 7.1, the eigenvalues for modes I and II fractures are insensitive to the electric boundary conditions as proven in Appendix B. Under the impermeable condition, only $u_{z}$ and $\phi$ are non-zero for mode III and mode IV, respectively. Under the permeable condition, $u_{z}$ and $\phi$ are coupled. Mode IV is taken to be the one dominated by nonzero $\phi$.

With $y$ taken to be the poling direction, Tables 7 and 8 list the computed eigenvalues for PZT-4 and cadmium sulfide, respectively. For the chosen poling direction, the governing equation and boundary condition for $u_{z}$ are independent of that for $u_{x}, u_{y}$ and $\phi$ as well as the electric boundary condition as discussed in Appendix C. Hence, the eigen-solution of mode III is insensitive to the electric boundary condition.

The mode IV singularities is dominated by non-zero electric potential and is the result of electric flux concentration at the crack tip. The concentration effect and thus the singularity strength drops with the relative permittivity (w.r.t. air) of the piezoelectrics. As the relative permittivities of PZT-4 and cadmium sulfide are respectively $\approx 550$ and $\approx 9$, it can be seen in Tables 5 to 8 that the mode IV singularity strength of the former is markedly stronger than that of the latter under the permeable condition. Noting that the strength reduces as the crack or notch angle reduces, the impermeable condition does not appear to be a realistic assumption for both PZT-4 and cadmium sulfide. Interestingly, the conducting condition is more realistic than the impermeable condition for cadmium sulfide. These findings are of use when nonlinear electric phenomena such as domian switching and electric saturation are considered.

Table 5. Eigenvalues for permeable and impermeable notch-cracks in PZT-4 poled along z-axis, see Figure 2

\begin{tabular}{|c|c|c|c|c|}
\hline notch angle $\alpha$ & electric b.c. & modes I \& II & mode III & mode IV \\
\hline \multirow{2}{*}{$0.1^{\mathrm{o}}$} & impermeable & $-0.500000,-0.499722$ & -0.499861 & -0.499861 \\
& permeable & ditto & -0.499861 & -0.245163 \\
\hline \multirow{2}{*}{$0.3^{\circ}$} & impermeable & $-0.500000,-0.499165$ & -0.499583 & -0.499583 \\
& permeable & ditto & -0.499583 & -0.374502 \\
\hline \multirow{2}{*}{$0.5^{\circ}$} & impermeable & $-0.500000,-0.498607$ & -0.499305 & -0.499305 \\
& permeable & ditto & -0.499305 & -0.416349 \\
\hline \multirow{2}{*}{$1.0^{\circ}$} & impermeable & $-0.500000,-0.497207$ & -0.498607 & -0.498607 \\
\cline { 3 - 5 } $2.0^{\circ}$ & permeable & ditto & -0.498607 & -0.453519 \\
\hline & impermeable & $-0.500000,-0.494382$ & -0.497207 & -0.497207
\end{tabular}




\begin{tabular}{|c|c|c|c|c|}
\hline & permeable & ditto & -0.497207 & -0.473622 \\
\hline \multirow{2}{*}{$3.0^{\circ}$} & impermeable & $-0.500000,-0.491527$ & -0.495798 & -0.495798 \\
& permeable & ditto & -0.495798 & -0.479828 \\
\hline
\end{tabular}

Table 6. Eigenvalues for permeable and impermeable notch cracks in cadmium sulfide poled along z-axis, see Figure 2

\begin{tabular}{|c|c|c|c|c|}
\hline notch angle $\alpha$ & electric b.c. & modes I \& II & mode III & mode IV \\
\hline \multirow{2}{*}{$0.1^{\circ}$} & impermeable & $-0.500000,-0.499722$ & -0.499861 & -0.499861 \\
& permeable & ditto & -0.499861 & -0.002314 \\
\hline \multirow{2}{*}{$0.3^{\circ}$} & impermeable & $-0.500000,-0.499165$ & -0.499583 & -0.499583 \\
& permeable & ditto & -0.499583 & -0.006909 \\
\hline \multirow{2}{*}{$0.5^{\circ}$} & impermeable & $-0.500000,-0.498607$ & -0.499305 & -0.499305 \\
& permeable & ditto & -0.499305 & -0.011459 \\
\hline \multirow{2}{*}{$1.0^{\circ}$} & impermeable & $-0.500000,-0.497207$ & -0.498607 & -0.498607 \\
& permeable & ditto & -0.498607 & -0.022619 \\
\hline \multirow{2}{*}{$2.0^{\circ}$} & impermeable & $-0.500000,-0.494382$ & -0.497207 & -0.497207 \\
& permeable & ditto & -0.497207 & -0.043961 \\
\hline \multirow{2}{*}{$3.0^{\circ}$} & impermeable & $-0.500000,-0.491528$ & -0.495798 & -0.495798 \\
& permeable & ditto & -0.495798 & -0.063918 \\
\hline
\end{tabular}

Table 7. Eigenvalues for permeable and impermeable notch cracks in PZT-4 poled along y-axis, see

Figure 2

\begin{tabular}{|c|c|c|c|c|}
\hline notch angle $\alpha$ & electric b.c. & modes I \& II & mode III & mode IV \\
\hline \multirow{2}{*}{$0.1^{\circ}$} & impermeable & $-0.500000,-0.499691$ & -0.499848 & -0.499841 \\
& permeable & $-0.500000,-0.499691$ & ditto & -0.227763 \\
\hline \multirow{2}{*}{$0.3^{\circ}$} & impermeable & $-0.500000,-0.499071$ & -0.499544 & -0.499524 \\
& permeable & $-0.500000,-0.499071$ & ditto & -0.361369 \\
\hline \multirow{2}{*}{$0.5^{\circ}$} & impermeable & $-0.500000,-0.498450$ & -0.499240 & -0.499206 \\
& permeable & $-0.500000,-0.498450$ & ditto & -0.406587 \\
\hline \multirow{2}{*}{$1.0^{\circ}$} & impermeable & $-0.500000,-0.496892$ & -0.498477 & -0.498410 \\
& permeable & $-0.500000,-0.496892$ & ditto & -0.447569 \\
\hline \multirow{2}{*}{$2.0^{\circ}$} & impermeable & $-0.500000,-0.493746$ & -0.496944 & -0.496809 \\
& permeable & $-0.500000,-0.493746$ & ditto & -0.470061 \\
\hline \multirow{2}{*}{$3.0^{\circ}$} & impermeable & $-0.499998,-0.490562$ & -0.495403 & -0.495198 \\
& permeable & $-0.499998,-0.490562$ & ditto & -0.477048 \\
\hline
\end{tabular}

Table 8. Eigenvalues for permeable and impermeable notch-cracks in cadmium sulfide (y being the poling direction), see Figure 2

\begin{tabular}{|c|c|c|c|c|}
\hline notch angle $\alpha$ & electric b.c. & modes I \& II & mode III & mode IV \\
\hline \multirow{2}{*}{$0.1^{\circ}$} & impermeable & $-0.500008,-0.499677$ & -0.499855 & -0.499864 \\
& permeable & $-0.500008,-0.499677$ & ditto & -0.002383 \\
\hline \multirow{2}{*}{$0.3^{\circ}$} & impermeable & $-0.500008,-0.499037$ & -0.499566 & -0.499592 \\
& permeable & $-0.500008,-0.499037$ & ditto & -0.007114 \\
\hline \multirow{2}{*}{$0.5^{\circ}$} & impermeable & $-0.500008,-0.498395$ & -0.499276 & -0.499320 \\
& permeable & $-0.500008,-0.498395$ & ditto & -0.011795 \\
\hline \multirow{2}{*}{$1.0^{\circ}$} & impermeable & $-0.500008,-0.496783$ & -0.498550 & -0.498639 \\
& permeable & $-0.500008,-0.496783$ & ditto & -0.023274 \\
\hline \multirow{2}{*}{$2.0^{\circ}$} & impermeable & $-0.500007,-0.493529$ & -0.497091 & -0.497270
\end{tabular}




\begin{tabular}{|c|c|c|c|c|}
\hline & permeable & $-0.500007,-0.493529$ & ditto & -0.045196 \\
\hline \multirow{2}{*}{$3.0^{\circ}$} & impermeable & $-0.500006,-0.490235$ & -0.495624 & -0.495895 \\
& permeable & $-0.500006,-0.490235$ & ditto & -0.065654 \\
\hline
\end{tabular}

Restricting ourselves to transverse isotropic piezoelectrics, it has been proven in Appendices B and $\mathrm{C}$ as well as reproduced by the computational results that modes I and II for $\mathrm{z}$ being the poling direction as well as mode III for y being the poling direction are insensitive to the electric boundary condition. Note worthily, Tables 5 to 8 also show that model III for $\mathrm{z}$ being the poling direction as well as modes I and II for y being the poling direction appear to be insensitive to the electric boundary condition. To further investigate these analytically unproven observations, more significant figures are considered. Table 9 lists the computed eigenvalues given in twelve significant figures for PZT-4 (remark : all computations are conducted in double precision that employs sixteen significant figures). For the eigenvalues that have been analytically proven to be insensitive to the electric boundary condition, the computed values are indistinguishable judging from the first ten significant figures. For the eigenvalues that cannot be analytically proven to be insensitive to the electric boundary condition (i.e. mode III for $\mathrm{z}$ being the poling direction as well as modes I and II for y being the poling direction), discrepancies can be observed in the seventh to ninth significant figures when the notch angle increases to $30^{\circ}$. These suggest that the considered eigenvalues for modes I, II \& III are highly insusceptible to the electric boundary condition.

Table 9. Eigenvalues given in twelve significant figures for notch-cracks in PZT-4

\begin{tabular}{|c|c|c|c|c|}
\hline $\begin{array}{l}\text { notch } \\
\text { angle, } \alpha\end{array}$ & $\begin{array}{l}\text { poling } \\
\text { direction }\end{array}$ & $\begin{array}{l}\text { electric } \\
\text { b.c. }\end{array}$ & modes I \& II & mode III \\
\hline \multirow[t]{2}{*}{$0.3^{\circ}$} & $\mathrm{Z}$ & $\begin{array}{l}\text { impermeable } \\
\text { permeable }\end{array}$ & $\begin{array}{l}-0.499999998571,-0.499165275738 \\
-0.499999998570,-0.499165275740\end{array}$ & $\begin{array}{l}-0.499582985251 \\
-0.499582985257\end{array}$ \\
\hline & $\mathrm{y}$ & $\begin{array}{l}\text { impermeable } \\
\text { permeable }\end{array}$ & $\begin{array}{l}-0.500000142036,-0.499071499935 \\
-0.500000142045,-0.499071499928\end{array}$ & $\begin{array}{l}-0.499544041035 \\
-0.499544041035\end{array}$ \\
\hline \multirow[t]{2}{*}{$3^{\circ}$} & $\mathrm{Z}$ & $\begin{array}{c}\text { impermeable } \\
\text { permeable }\end{array}$ & $\begin{array}{l}-0.499998571858,-0.491526882698 \\
-0.499998571858,-0.491526882697\end{array}$ & $\begin{array}{l}-0.495798318752 \\
-0.495798318689 \\
\end{array}$ \\
\hline & $\mathrm{y}$ & $\begin{array}{l}\text { impermeable } \\
\text { permeable }\end{array}$ & $\begin{array}{l}-0.499998029229,-0.490561666113 \\
-0.499998029236,-0.490561666115\end{array}$ & $\begin{array}{l}-0.495402889654 \\
-0.495402889654\end{array}$ \\
\hline \multirow[t]{2}{*}{$30^{\circ}$} & $\mathrm{Z}$ & $\begin{array}{l}\text { impermeable } \\
\text { permeable }\end{array}$ & $\begin{array}{l}-0.498546991231,-0.401808148947 \\
-0.498546991231,-0.401808148948\end{array}$ & $\begin{array}{l}-0.454545453924 \\
-0.454545454066\end{array}$ \\
\hline & $\mathrm{y}$ & $\begin{array}{l}\text { impermeable } \\
\text { permeable }\end{array}$ & $\begin{array}{l}-0.497894065285,-0.389059871202 \\
-0.497894085474,-0.389059874432\end{array}$ & $\begin{array}{l}-0.450119970024 \\
-0.450119970024 \\
\end{array}$ \\
\hline
\end{tabular}




\section{CLOSURE}

An one-dimensional finite element procedure is formulated for determining the eigen-solutions of the electromechanical field problem. The procedure is validated by comparing its predictions with other established results and employed to study permeable notches which has aroused considerable attention (Dunn 1994; Hao \& Shen 1994). For small crack or notch angle, it is noted that the impermeable condition does not appear to be realistic. These findings are of interest when nonlinear electric phenomena such as domian switching and electric saturation are considered.

Compared to various analytical approaches, the developed procedure is versatile and can readily be applied to study the eigensolutions for a large variety of configurations such as piezoelectrics/ piezoelectrics and piezoelectric/metal interfaces in bimorph and multi-stack actuators.

Acknowledgment - The work described in this paper was supported by a grant from the Research Grant Council of the Hong Kong (CERG Project No. HKU 7083/00E). Part of the work was completed when the third author, H.Fan, was visiting the University of Hong Kong as a William Mong Visiting Research Fellow. 


\section{REFERENCES}

Anderson, T.L. 1995. Fracture Mechanics - Fundamentals and Applications. $2^{\text {nd }}$ Edn., CRC Press, London.

Bazant, Z.P., Estenssoro, L.F., 1979. Surface singularity and crack propagation. Int. J.Solids \& Structures 15, 405-426.

Benthem, J.P., 1980. The quarter-infinite crack in a half-space; alternative and additional solutions. Int. J.Solids \& Structures 16, 119-130.

Dunn, M.L., 1994. The effects of crack face boundary conditions on the fracture mechanics of piezoelectric solid. Engrg.Fract.Mech. 48, 25-39.

Gu, L., Belytschko, T., 1994. A numerical study of stress singularities in a two-material wedge. Int. J.Solids \& Structures 31, 865-889.

Hao, T.-H., Shen, Z.-Y., 1994. A new electric boundary condition of electric fracture mechanics and its applications. Engrg.Fract.Mech. 47, 793-803.

Hartranft, R.J., Sih, G.C., 1969. The use of eigenfunction expansions in the general solution of three dimensional crack problems. J.Math.Mech. 19, 123-138.

Kuo, C.-M., Barnett, D.M., 1991. Stress singularities of interfacial cracks in bonded piezoelectric half-spaces. In: Wu, J.J., Ting, T.C.T., Barnett, D.M. (Eds.), Modern Theory of Anisotropic Elasticity and Applications, SIAM, Philadelphia, pp.33-50.

Mcmeeking, R.M., 1989. Electrostrictive stresses near crack-like flaws. J.Appl.Math. \& Phy. 40, 615-627.

Pageau, S.S., Biggers, S.B., 1995. Finite element evaluation of free-edge singular stress fields in anisotropic materials. Inter.J.Numer.Methods Engng. 38, 2225-2239

Pageau, S.S., Biggers, S.B., 1996. A finite element approach to three-dimensional singular stress states in anisotropic multi-material wedges and junctions. Int.J.Solids and Structures 33, 33-47.

Pak, Y.E., 1990. Crack extension force in a piezoelectric material. J.Appl.Mech 57, 647-653.

Park, S.B., Sun, C.T., 1995. Effect of electric field on fracture of piezoelectric ceramics. Int.J.Fract 70, 203-216.

Parton, V.Z., 1976. Fracture mechanics of piezoelectric materials. Acta Astronautica 3, 671-683.

Qin, Q.-H., Yu, S.-W., 1997. An arbitrary-orientated plane crack terminating at the interface between dissimilar piezoelectric materials. Int.J.Solids and Structures 34, 581-590.

Sosa, H.A., Pak, Y.E., 1990. Three-dimensional eigenfunction analysis of a crack in a piezoelectric material. Int.J.Solids and Structures 26, 1-15.

Sosa, H., 1992. On the fracture mechanics of piezoelectric solids. Int.J.Solids and Structures 29, 2613-2622.

Suo, Z., Kuo, C.-M., Barnett, D.M., Willis, J.R., 1992. Fracture mechanics for piezoelectric ceramics. J.Mech.Phys.Solids 40, 739-765.

Sze, K.Y., Wang, H.-T., 2000. A simple finite element formulation for computing stress singularities at bimaterial interfaces. Finite Elements in Analysis \& Design 35, 97-118.

Yamada, Y., Okumura, H., 1983. Finite element analysis of stress and strain singularity eigenstate in inhomogeneous media or composite materials. In: Atluri, S.N., Gallagher, R.H., Zienkiewicz, O.C. (Eds.), Hybrid \& Mixed Finite Element Methods, Wiley, Chichester, pp.325-343.

Yosibash, Z., Szabo, B.A., 1995. Numerical analysis of singularities in two-dimensions part 1: computation of eigenpairs. Inter.J.Numer.Methods Engng., 38, 2055-2082. 


\section{APPENDIX A : Divergence Theorems for Prismatic Sectorial Domain}

It is trivial to show that

$$
\frac{1}{r} \delta \mathbf{u}^{T}\left[\boldsymbol{\nabla}_{m}(r \boldsymbol{\sigma})\right]+\delta \boldsymbol{\epsilon}^{T} \boldsymbol{\sigma}=\frac{1}{r} \boldsymbol{\nabla}\left\{\begin{array}{l}
r \delta \mathbf{u}^{T} \boldsymbol{\sigma}_{r} \\
r \delta \mathbf{u}^{T} \boldsymbol{\sigma}_{\theta} \\
r \delta \mathbf{u}^{T} \boldsymbol{\sigma}_{z}
\end{array}\right\}, \frac{1}{r} \delta \phi \boldsymbol{\nabla}(r \mathbf{D})=\frac{1}{r} \boldsymbol{\nabla}(r \delta \phi \mathbf{D})+\delta \mathbf{E}^{T} \mathbf{D}
$$

By integrating the above identities over the sectorial prism $\Omega$ defined by $r \in[0, R], \theta \in\left[\theta_{S}, \theta_{T}\right]$ and $z \in[0, L]$ as shown in Figure 6, the following divergence theorems can be obtained :

$$
\begin{aligned}
\int_{\Omega}\left\{\frac{1}{r} \delta \mathbf{u}^{T}\right. & {\left.\left[\nabla_{m}(r \boldsymbol{\sigma})\right]+\delta \boldsymbol{\epsilon}^{T} \boldsymbol{\sigma}\right\} d \Omega } \\
& =\left.R \int_{\theta_{S}}^{\theta_{T}} \int_{0}^{L}\left(\delta \mathbf{u}^{T} \boldsymbol{\sigma}_{r}\right)\right|_{r=R} d z d \theta+\left.\int_{0}^{R} \int_{0}^{L}\left(\delta \mathbf{u}^{T} \boldsymbol{\sigma}_{\theta}\right)\right|_{\theta=\theta_{S}} ^{\theta=\theta_{T}} d z d r+\left.\int_{0}^{R} \int_{\theta_{S}}^{\theta_{T}}\left(r \delta \mathbf{u}^{T} \boldsymbol{\sigma}_{z}\right)\right|_{z=0} ^{z=L} d r d \theta
\end{aligned}
$$

and

$$
\begin{aligned}
\int_{\Omega}\left[\frac{1}{r} \delta \phi \boldsymbol{\nabla}\right. & \left.(r \mathbf{D})-\delta \mathbf{E}^{T} \mathbf{D}\right] d \Omega \\
& =R \int_{\theta_{S}}^{\theta_{T}} \int_{0}^{L}\left(\delta \phi D_{r}\right) d \theta d z+\left.\int_{0}^{R} \int_{0}^{L}\left(\delta \phi D_{\theta}\right)\right|_{\theta=\theta_{S}} ^{\theta=\theta_{T}} d r d z+\left.\int_{0}^{R} \int_{\theta_{S}}^{\theta_{T}}\left(r \delta \phi D_{z}\right)\right|_{z=0} ^{z=L} d r d \theta
\end{aligned}
$$

\section{APPENDIX B : Poling Direction Parallel to z-axis}

The following discussion is restricted to transverse isotropic materials with its principal material directions 1, 2 and 3 (poling direction) parallel to the $\mathrm{x}-, \mathrm{y}$ - and $\mathrm{z}$-directions, respectively. Noting that the displacement and electric potential are assumed to be independent of $z$, we have

$$
\begin{aligned}
& \boldsymbol{\epsilon}=\left\{\varepsilon_{x}, \varepsilon_{y}, \varepsilon_{z}, \gamma_{y z}, \gamma_{z x}, \gamma_{x y}\right\}^{T}=\left\{u_{x},_{x}, u_{y},_{y}, 0, u_{z},_{y}, u_{z},_{x}, u_{x},_{y}+u_{y},_{x}\right\}^{T}, \\
& \mathbf{E}=\left\{E_{x}, E_{y}, E_{z}\right\}^{T}=\left\{-\phi,_{x},-\phi,_{y}, 0\right\}^{T}
\end{aligned}
$$

With $z$-axis aligned with the third material principal (poling) direction, the constitutive relation for transverse isotropic piezoelectrics can be expressed as :

$$
\left\{\begin{array}{c}
\sigma_{x} \\
\sigma_{y} \\
\sigma_{z} \\
\tau_{y z} \\
\tau_{z x} \\
\tau_{x y}
\end{array}\right\}=\left[\begin{array}{cccccc}
c_{11} & c_{12} & c_{13} & 0 & 0 & 0 \\
c_{12} & c_{11} & c_{13} & 0 & 0 & 0 \\
c_{13} & c_{13} & c_{33} & 0 & 0 & 0 \\
0 & 0 & 0 & c_{44} & 0 & 0 \\
0 & 0 & 0 & 0 & c_{44} & 0 \\
0 & 0 & 0 & 0 & 0 & \left(c_{11}-c_{12}\right) / 2
\end{array}\right]\left\{\begin{array}{c}
u_{x},_{x} \\
u_{y},{ }_{y} \\
0 \\
u_{z},_{y} \\
u_{z},,_{x} \\
u_{x},{ }_{y}+u_{y},{ }_{x}
\end{array}\right\}+\left[\begin{array}{ccc}
0 & 0 & e_{31} \\
0 & 0 & e_{31} \\
0 & 0 & e_{33} \\
0 & e_{15} & 0 \\
e_{15} & 0 & 0 \\
0 & 0 & 0
\end{array}\right]\left\{\begin{array}{c}
\phi,_{x} \\
\phi,,_{y} \\
0
\end{array}\right\},
$$




$$
\left\{\begin{array}{l}
D_{x} \\
D_{y} \\
D_{z}
\end{array}\right\}=\left[\begin{array}{cccccc}
0 & 0 & 0 & 0 & e_{15} & 0 \\
0 & 0 & 0 & e_{15} & 0 & 0 \\
e_{31} & e_{31} & e_{33} & 0 & 0 & 0
\end{array}\right]\left\{\begin{array}{c}
u_{x},_{x} \\
u_{y},{ }_{y} \\
0 \\
u_{z},{ }_{y} \\
u_{z},,_{x} \\
u_{x},{ }_{y}+u_{y}, x
\end{array}\right\}-\left[\begin{array}{ccc}
\epsilon_{11} & 0 & 0 \\
0 & \epsilon_{11} & 0 \\
0 & 0 & \in_{33}
\end{array}\right]\left\{\begin{array}{c}
\phi,_{x} \\
\phi, y_{y} \\
0
\end{array}\right\}
$$

from which the governing equations (stress equilibrium and charge balance conditions) become :

$$
\begin{aligned}
& \sigma_{x},_{x}+\tau_{x y},_{y}+\tau_{z x},_{z}=0 \Rightarrow c_{11} u_{x},_{x x}+c_{12} u_{y},_{x y}+\left(c_{11}-c_{12}\right)\left(u_{x},_{y y}+u_{y},_{x y}\right) / 2=0 \text {, } \\
& \tau_{x y},_{x}+\sigma_{y},{ }_{y}+\tau_{y z},_{z}=0 \Rightarrow\left(c_{11}-c_{12}\right)\left(u_{x},_{x y}+u_{y},{ }_{x x}\right) / 2+c_{12} u_{x},_{x y}+c_{11} u_{y},{ }_{y y}=0 \text {, } \\
& \tau_{z x},{ }_{x}+\tau_{y z},{ }_{y}+\sigma_{z},{ }_{z}=0 \Rightarrow\left(\partial^{2} / \partial x^{2}+\partial^{2} / \partial y^{2}\right) \tilde{u}_{z}=0 \text {, } \\
& D_{x},_{x}+D_{y},_{y}+D_{z},{ }_{z}=0 \Rightarrow\left(\partial^{2} / \partial x^{2}+\partial^{2} / \partial y^{2}\right) \tilde{\phi}=0
\end{aligned}
$$

where $\tilde{u}_{z}=u_{z}+e_{15} \phi / c_{44}$ and $\tilde{\phi}=\phi-e_{15} u_{z} / \epsilon_{11}$. On the other hand, the traction-free boundary conditions and charge density $D_{n}$ at the crack/notch surfaces whose unit outward normal is $\left\{n_{x}, n_{y}\right.$, $\left.n_{z}\right\}^{T}=\left\{n_{x}, n_{y}, 0\right\}^{T}$ are :

$$
\begin{aligned}
& t_{x}=n_{x} \sigma_{x}+n_{y} \tau_{x y}=n_{x}\left(c_{11} u_{x},{ }_{x}+c_{12} u_{y},{ }_{y}\right)+n_{y}\left(c_{11}-c_{12}\right)\left(u_{x},{ }_{y}+u_{y},_{x}\right) / 2=0, \\
& t_{y}=n_{x} \tau_{x y}+n_{y} \sigma_{y}=n_{x}\left(c_{11}-c_{12}\right)\left(u_{x},{ }_{y}+u_{y},_{x}\right) / 2+n_{y}\left(c_{12} u_{x},{ }_{x}+c_{11} u_{y},{ }_{y}\right)=0, \\
& t_{z}=n_{x} \tau_{z x}+n_{y} \tau_{y z}=c_{44}\left(n_{x} \tilde{u}_{z},,_{x}+n_{y} \tilde{u}_{z},{ }_{y}\right)=0, D_{n}=n_{x} D_{x}+n_{y} D_{y}=-\epsilon_{11}\left(n_{x} \tilde{\phi},_{x}+n_{y} \tilde{\phi},{ }_{y}\right)
\end{aligned}
$$

The following points can be drawn from Eqn.(A3) and Eqn.(A4) :

- Regardless of the electric boundary conditions, the governing equations and boundary conditions for $u_{x}$ and $u_{y}$ are independent of that for $\tilde{u}_{z}$ and $\tilde{\phi}$. The two eigen solutions arising from $u_{x}$ and $u_{y}$ represent the conventional mode I and mode II fractures.

- For impermeable cracks/notches where $D_{n}=0, \tilde{u}_{z}$ and $\tilde{\phi}$ are subjected to the same governing and boundary conditions. Therefore, the two eigen solutions arising from them are identical. The corresponding eigen functions can be expressed as one with $u_{z}=0$ and one with $\phi=0$. While the latter represents the conventional mode III fracture, the former is commonly known as the mode IV fracture (Suo et al 1992).

- For conducting cracks/notches, the radial derivative of $\phi$ cannot be singular. Thus, $\phi$ in all physical singular eigenmodes vanishes and $\tilde{u}_{z}$ reduces to $u_{z}$. Again, the eigen solution arising from $u_{z}$ represents the conventional mode III fracture. 


\section{APPENDIX C : Poling Direction Perpendicular to z-axis}

Without losing generality, we will assume that $\mathrm{y}$ is the poling direction. The constitutive relation analogous to Eqn.(A2) become :

$$
\begin{aligned}
& \left\{\begin{array}{c}
\sigma_{z} \\
\sigma_{x} \\
\sigma_{y} \\
\tau_{x y} \\
\tau_{y z} \\
\tau_{z x}
\end{array}\right\}=\left[\begin{array}{cccccc}
c_{11} & c_{12} & c_{13} & 0 & 0 & 0 \\
c_{12} & c_{11} & c_{13} & 0 & 0 & 0 \\
c_{13} & c_{13} & c_{33} & 0 & 0 & 0 \\
0 & 0 & 0 & c_{44} & 0 & 0 \\
0 & 0 & 0 & 0 & c_{44} & 0 \\
0 & 0 & 0 & 0 & 0 & \left(c_{11}-c_{12}\right) / 2
\end{array}\right]\left\{\begin{array}{c}
0 \\
u_{x},,_{x} \\
u_{y},_{y} \\
u_{x},{ }_{y}, u_{y},,_{x} \\
u_{z},{ }_{y} \\
u_{z},{ }_{x}
\end{array}\right\}+\left[\begin{array}{ccc}
0 & 0 & e_{31} \\
0 & 0 & e_{31} \\
0 & 0 & e_{33} \\
0 & e_{15} & 0 \\
e_{15} & 0 & 0 \\
0 & 0 & 0
\end{array}\right]\left\{\begin{array}{c}
0 \\
\phi,_{x} \\
\phi,,_{y}
\end{array}\right\}, \\
& \left\{\begin{array}{l}
D_{z} \\
D_{x} \\
D_{y}
\end{array}\right\}=\left[\begin{array}{cccccc}
0 & 0 & 0 & 0 & e_{15} & 0 \\
0 & 0 & 0 & e_{15} & 0 & 0 \\
e_{31} & e_{31} & e_{33} & 0 & 0 & 0
\end{array}\right]\left\{\begin{array}{c}
0 \\
u_{x},{ }_{x} \\
u_{y},{ }_{y} \\
u_{x},{ }_{y}+u_{y},{ }_{x} \\
u_{z},{ }_{y} \\
u_{z},{ }_{x}
\end{array}\right\}-\left[\begin{array}{ccc}
\epsilon_{11} & 0 & 0 \\
0 & \in_{11} & 0 \\
0 & 0 & \in_{33}
\end{array}\right]\left\{\begin{array}{c}
0 \\
\phi,,_{x} \\
\phi,,_{y}
\end{array}\right\}
\end{aligned}
$$

By expressing the stress equilibrium condition, charge balance condition and the crack face conditions in terms of the displacement components (which are independent of $\mathrm{z}$ ), it is trivial to show that $\left(u_{x}, u_{y}, \phi\right)$ and $u_{z}$ are uncoupled regardless of the electric boundary condition. Moreover, $u_{x}, u_{y}$ and $\phi$ vanish in the eigen solution arising from $u_{z}$. 


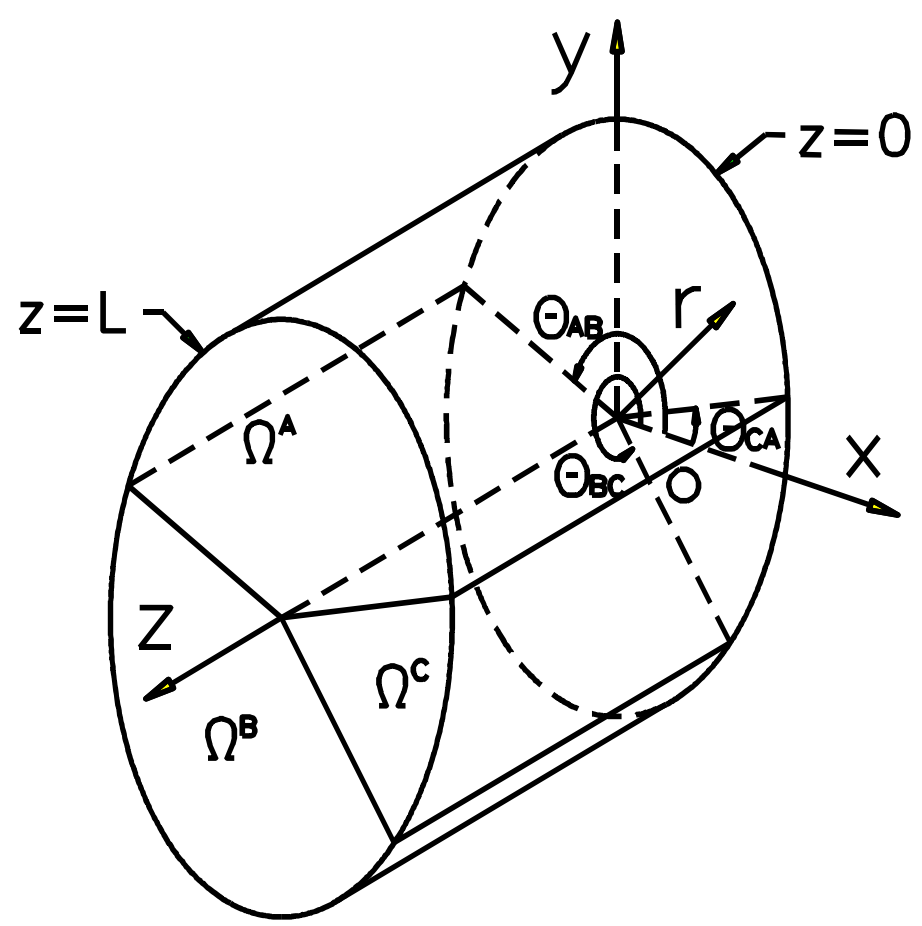

Figure 1. A sectorial prismatic domain with three subdomains

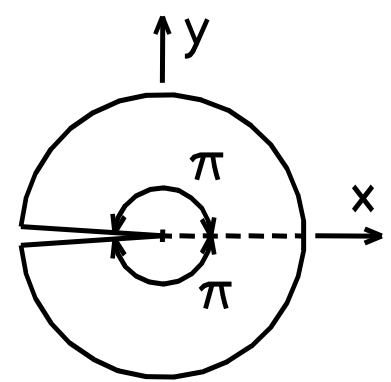

(a)

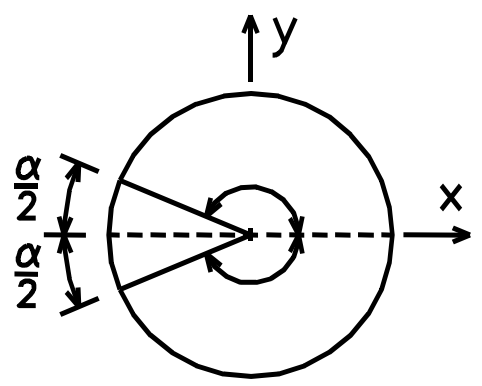

(b)

Figure 2. (a) Crack modelled by two piezoelectric elements, (b) permeable notch of notch angle $\alpha$ modelled by two piezoelectric and two air elements. 


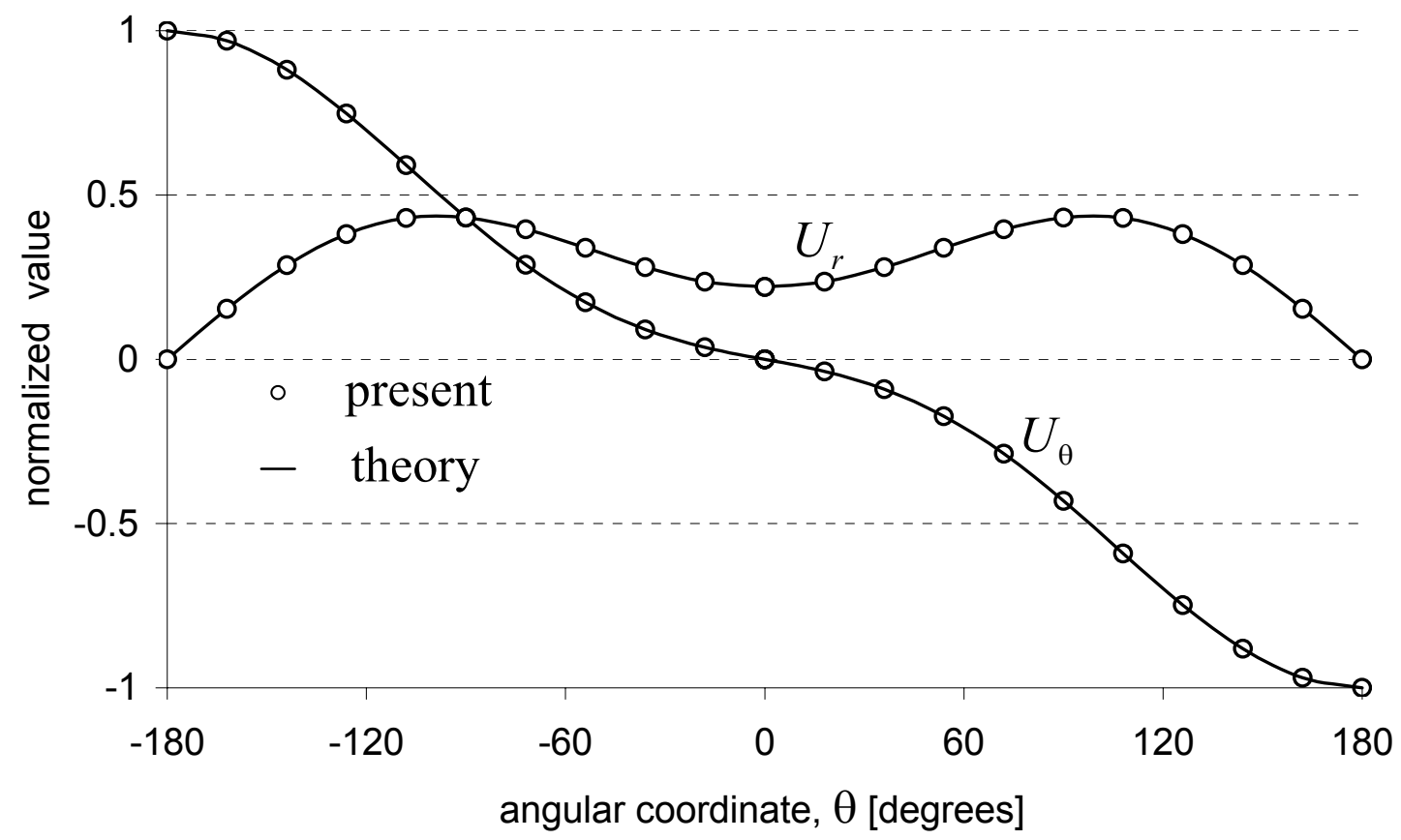

Figure 3. Normalized radial and circumference displacements for mode I fracture.

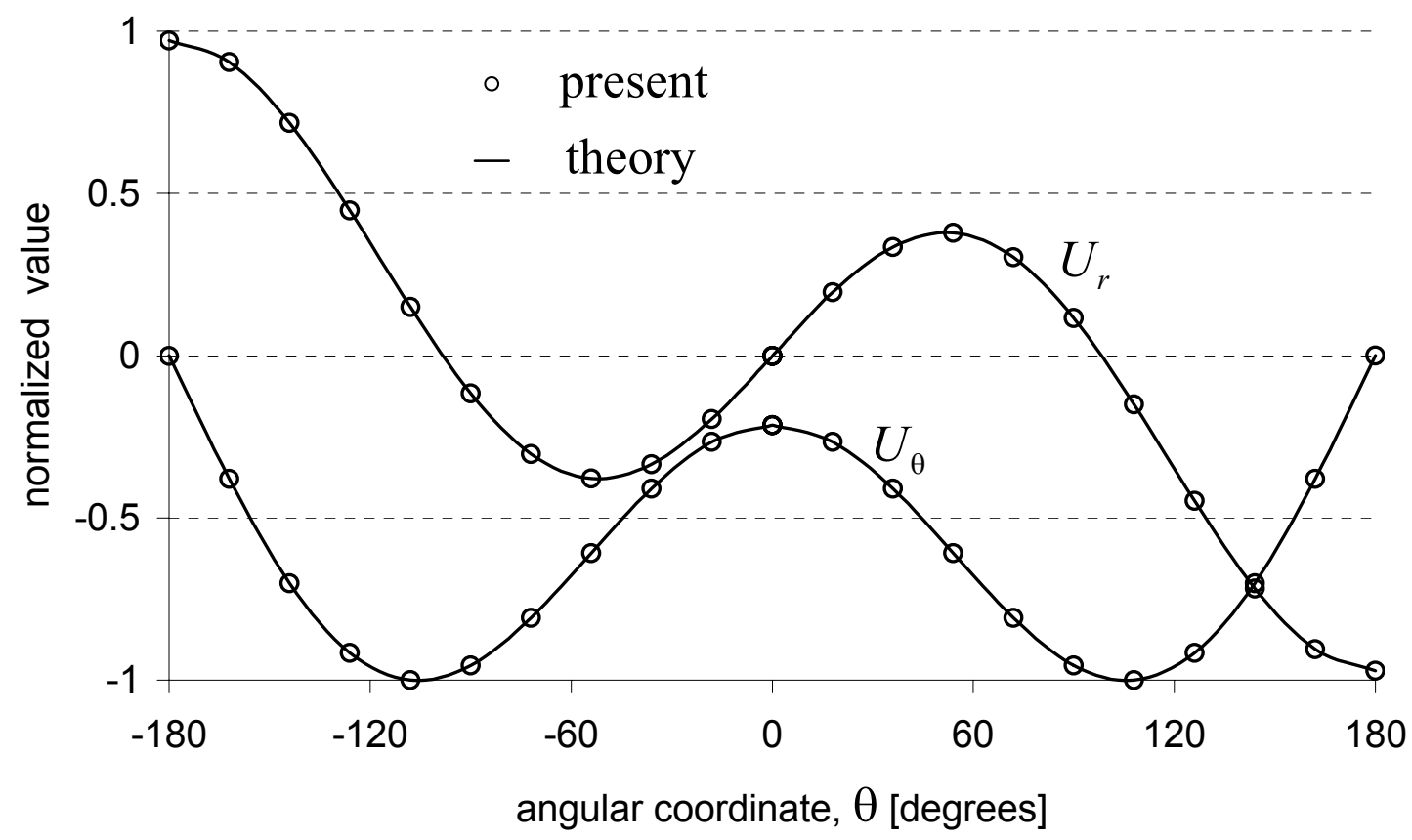

Figure 4. Normalized radial and circumference displacements for mode II fracture. 


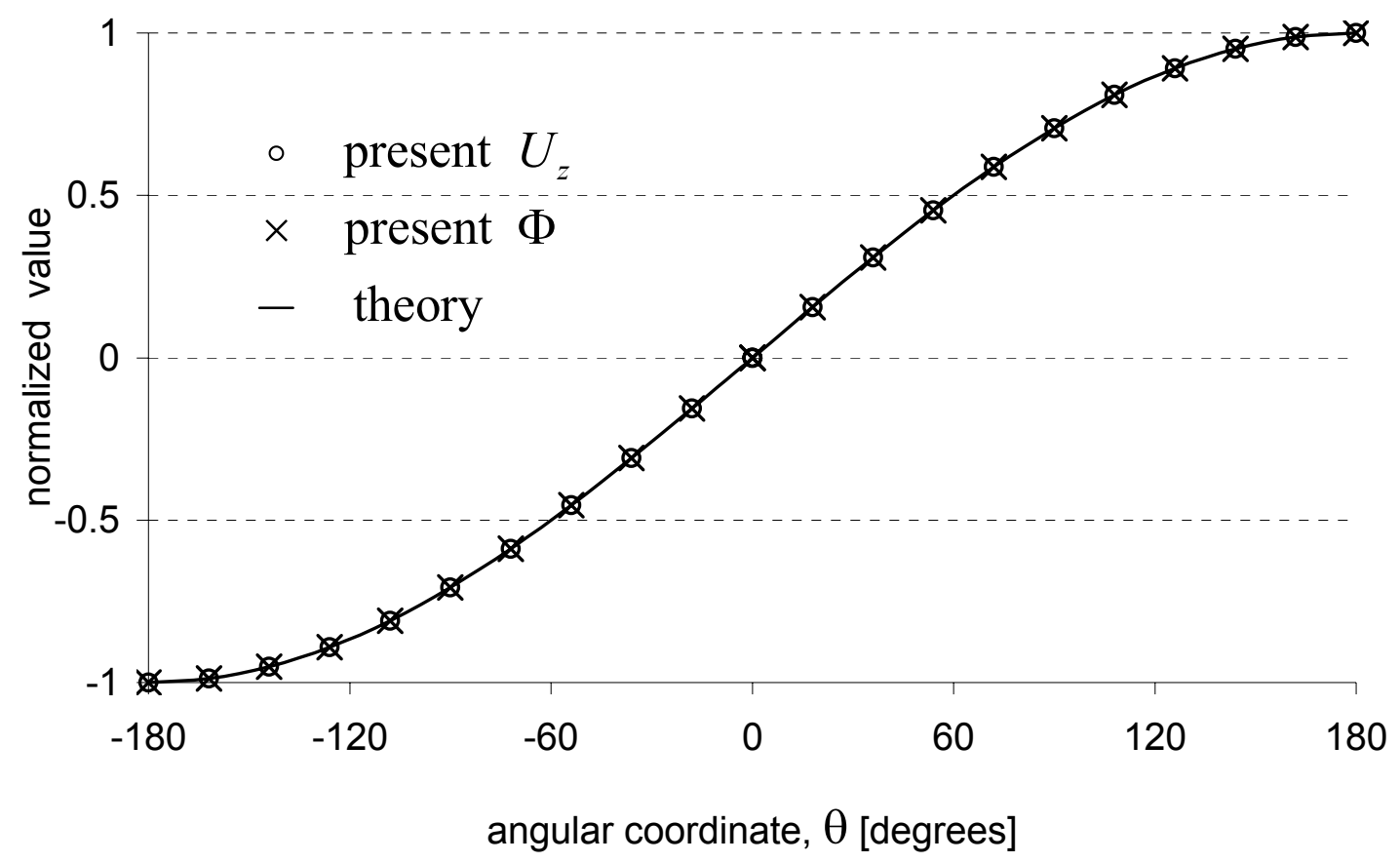

Figure 5. Normalized longitudinal displacement for mode III fracture and normalized electric potential for mode IV fracture.

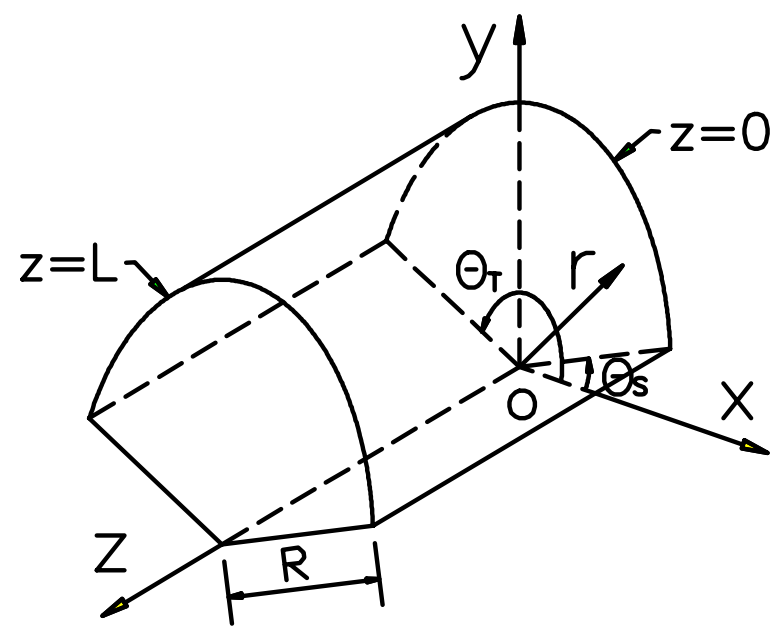

Figure 6. A sectorial prism. 